EPJ manuscript No.

(will be inserted by the editor)

\title{
Final Results from phase II of the Mainz Neutrino Mass Search in Tritium $\beta$ Decay
}

\author{
Ch. Kraus ${ }^{1,8 a}$, B. Bornschein $1,2 a$, L. Bornschein ${ }^{1,3 a}$, J. Bonn ${ }^{1}$, B. Flatt ${ }^{1}$, A. Kovalik ${ }^{4}$, B. Ostrick ${ }^{1,6}$, E.W. Otten ${ }^{1 \mathrm{~b}}$ \\ J.P. Schall ${ }^{1}$, Th. Thümmler ${ }^{1,6}$, and Ch. Weinheimer ${ }^{1,5,7}$ \\ 1 Institut für Physik der Johannes Gutenberg-Universität Mainz, D-55099 Mainz, Germany \\ ' 2 present address: Forschungszentrum Karlsruhe, Tritiumlabor, D-76344 Eggenstein-Leopoldshafen, Germany \\ 3 present address: Universität Karlsruhe (TH), Institut für exp. Kernphysik, Postfach 6980, D-76128 Karlsruhe, Germany \\ 4 on leave from the Nuclear Physics Institute of the Acad. Sci. Czech Republic, CZ-25068 Rez near Prague \\ 5 Helmholtz-Institut für Strahlen und Kernphysik, Universität Bonn, D-53115 Bonn, Germany \\ 6 present address: Helmholtz-Institut für Strahlen und Kernphysik, Universität Bonn, D-53115 Bonn, Germany \\ 7 present address: Institut für Kernphysik, Universität Münster, D-48149 Münster, Germany \\ ' 8 present address: departement of physics, Queen's university, K7L3N6 Kingston, Canada
}

the date of receipt and acceptance should be inserted later

\begin{abstract}
The paper reports on the improved Mainz experiment on tritum $\beta$ spectroscopy which yields a 10 times' higher signal to background ratio than before. The main experimental effects and systematic uncertainties have been investigated in side experiments and possible error sources have been eliminated. Extensive data taking took place in the years 1997 to 2001. A residual analysis of the data sets yields for the square of the electron antineutrino mass the final result of $m^{2}\left(\nu_{e}\right)=\left(-0.6 \pm 2.2_{\text {stat }} \pm 2.1_{\text {syst }}\right) \mathrm{eV}^{2} / \mathrm{c}^{4}$. We derive an upper limit of $m\left(\nu_{e}\right) \leq 2.3 \mathrm{eV} / \mathrm{c}^{2}$ at $95 \%$ confidence level for the mass itself.
\end{abstract}

PACS. 1 460.Pq Neutrino mass and mixing - 23.40.- s Beta decay - 2930.Dn Electron spectroscopy- 2930.Aj Charged particle spectrometers: electric and magnetic

\section{Introduction}

In recent years observations of atmospheric, solar and reactor neutrinos 1,2,3,4,5,6,8,9 in large underground detectors have discovered and established strong mixing among the 3 neutrino generations $\nu_{1}, \nu_{2}, \nu_{3}$ produced in weak decays. The mixing manifests itself in neutrino flavour oscillations whose wave numbers are proportional to the differences of the squared masses $\Delta m_{i j}^{2}=\mid m^{2}\left(\nu_{i}\right)-$ $m^{2}\left(\nu_{j}\right) \mid$ of the mixing generations. Neutrino flavour eigenstates $\nu_{e}, \nu_{\mu}, \nu_{\tau}$ produced in weak interactions with electrons, muons or taus are thus connected to the mass eigenstates $\nu_{1}, \nu_{2}, \nu_{3}$ through a unitary mixing matrix $U$. So far oscillations $\nu_{e} \rightarrow \nu_{\mu}$ and $\nu_{\mu} \rightarrow \nu_{\tau}$ have been observed yielding mass differences $5.5 \cdot 10^{-5} \mathrm{eV}^{2} / \mathrm{c}^{4} \leq \Delta m_{12}^{2} \leq$ $1.9 \cdot 10^{-4} \mathrm{eV}^{2} / \mathrm{c}^{4}$ and $1.4 \cdot 10^{-3} \mathrm{eV}^{2} / \mathrm{c}^{4} \leq \Delta m_{23}^{2} \leq 6.0$. $10^{-3} \mathrm{eV}^{2} / \mathrm{c}^{4}$, taken from a recent combined analysis of oscillation parameters [10.

The fundamental discovery of finite mass differences between neutrino generations has re-stimulated the question about their absolute scale which is left open by any kind of interference experiment, necessarily. It could range

a This paper comprises principal parts of the $\mathrm{PhD}$ thesises of Christine Kraus, Beate Bornschein and Lutz Bornschein.

b corresponding author: e-mail: Ernst.Otten@uni-mainz.de from a hierarchical ordering with $m_{1}^{2}$ or $m_{3}^{2}$ being much smaller than either of the measured $\Delta m_{i j}^{2}$ values to a quasi degenerate situation where these differences are sitting on a much higher socket $m^{2} \gg \Delta m_{i j}^{2}$ (see e.g. [10]). Assuming $m_{1} \approx 0 \mathrm{eV} / \mathrm{c}^{2}$, the former case would yield $m_{2} \approx \sqrt{\Delta m_{12}^{2}} \approx 0.01 \mathrm{eV} / \mathrm{c}^{2}$ and $m_{3} \approx \sqrt{\Delta m_{23}^{2}} \approx 0.05$ $\mathrm{eV} / \mathrm{c}^{2}$. An experimental hint towards a degenerate solution came recently from a reanalysis 11 12 of earlier, and from new data of the Heidelberg Moscow experiment on neutrinoless double $\beta$ decay of ${ }^{76} \mathrm{Ge}$. If due to virtual emission and reabsorption of Majorana neutrinos the observed rate would correspond to a so-called effective neutrino mass

$$
m_{e e}=\left.\left|\sum m\left(\nu_{j}\right)\right| U_{e j}\right|^{2} e^{i \phi_{j}} \mid
$$

in the limits $0.1 \mathrm{eV} / \mathrm{c}^{2} \leq m_{e e} \leq 0.9 \mathrm{eV} / \mathrm{c}^{2}$ (99.7\% C.L.) 12. The $\phi$ are phase factors of the mixing matrix $U$. Although based on a $4 \sigma$ signal, this decay mode could still be modified by the exchange of some other non-standard particles.

Since in the universe a huge amount of about 336 relic neutrinos $/ \mathrm{cm}^{3}$ are supposed to be left over from the Big Bang, a sufficient rest mass could play an important role in the total mass balance, in particular as so-called hot dark matter during the early phase of cosmic evolution. Here the fine granulation of fluctuations, observed in the 
Ch. Kraus et al.: Final Results from the Mainz Neutrino Mass Experiment

temperature of the cosmic microwave background (CMB) as well as in the large scale structure of the distribution of galaxies (LSS), constrains the neutrino mass. Combined analyses of recent surveys yield upper mass limits of $0.23 \mathrm{eV} / \mathrm{c}^{2}$ 13] or somewhat more conservatively $0.33 \mathrm{eV} / \mathrm{c}^{2}$ 14,15 assuming 3 degenerate neutrino generations. Another analysis quotes a finite mass of $\sim 0.2 \mathrm{eV} / \mathrm{c}^{2}$ even [16. Still there is a caveat in this kind of analysis: it results from fitting a parametrized cosmological model in which $95 \%$ of the gravitational potential have to be attributed to unknown sources of matter and energy.

From the above discussion we conclude that a model independent, absolute mass measurement is indispensable even if the sensitivity limit of alternative, model dependent methods is not reached yet.

Among the model independent measurements, the investigation of the $\beta$ spectrum of tritium near its endpoint has yielded by far the most sensitive limits on the neutrino mass (strictly speaking the mass of the electron antineutrino) in the past. Until the early nineties magnetic $\beta$ spectrometers prevailed (reviewed in 17.18); thereafter electrostatic filters with magnetic adiabatic collimation (MAC-E-Filters) took over thanks to their higher transmission and resolution (reviewed recently in 19,20]). They were proposed and realized independently in Mainz 21] and Troitsk 22,23. Our spectrometer yielded first results in 1991 from which we have extracted an upper limit of $m\left(\nu_{e}\right)<7.2 \mathrm{eV} / \mathrm{c}^{2}$ (95\% C.L.) [25]; it improved to $5.6 \mathrm{eV} / \mathrm{c}^{2}$ at the end of phase I of this experiment [26]. These early results still suffered from small spectral distortions farer off the endpoint with a tendency to draw the observable $m^{2}\left(\nu_{e}\right)$ into the unphysical negative sector the more, the farer the spectral interval, used in the analysis, was extended below the endpoint.

After the reason for this effect had been identified, we performed in the years 1995-97 a substantial improvement program. It solved not only that problem but also improved the signal to background ratio by a factor of 10 . In 1997 we started phase II of running, yielding in the first year a limit down to $2.8 \mathrm{eV} / \mathrm{c}^{2}$ (95\% C.L.) 27] which was published in parallel to a $2.5 \mathrm{eV} / \mathrm{c}^{2}$ limit (95\% C.L.) from Troitsk 28. In the second year of data taking our value improved to $2.2 \mathrm{eV} / \mathrm{c}^{2}$ (95\% C.L.), communicated in 29. This limit was obtained from the experimental result $m^{2}\left(\nu_{e}\right)=\left(-1.6 \pm 2.5_{\text {stat }} \pm 2.1_{\text {sys }}\right) \mathrm{eV}^{2} / \mathrm{c}^{4}$. In the present paper we are giving a final report on this experiment, its analysis and its results.

The paper is organized as follows: In section 2 we resume briefly the principle of the experiment and discuss its sensitivity. In section 3 we describe the improvement program carried out for phase II. In section 4 we report on the data taking periods in the years 1997-2001. The data are analyzed and discussed in section 5 . In section 6 follows a discussion of the results. Conclusions and outlook are given in section 7 .

\section{$2 \beta$-spectrum and neutrino mass measured by an integrating electrostatic filter.}

\section{$2.1 \beta$-spectrum in $\mathrm{T}_{2}$ decay}

Since we observe only the kinetic energy $E$ of the $\beta$ particle we are measuring actually a sum of $\beta$ spectra, leading each with probability $P_{i}$ to a final state of excitation energy $V_{i}$ of the daughter and with probability $\left|U_{e j}\right|^{2}$ to a neutrino mass eigenstate $m\left(\nu_{j}\right)$. Hence the differential decay rate (Fig.1) is

$$
\begin{aligned}
\frac{\mathrm{d} R}{\mathrm{~d} E}= & N \frac{G_{f}^{2}}{2 \pi^{3} \hbar^{7} c^{5}} \cos ^{2}\left(\Theta_{c}\right)|M|^{2} F(E, \mathrm{Z}+1) \\
& p\left(E+m_{e} \mathrm{c}^{2}\right) \sum_{i j} P_{i}\left(E_{0}-V_{i}-E\right) \cdot \\
& \left|U_{e j}\right|^{2} \sqrt{\left(E_{0}-V_{i}-E\right)^{2}-m^{2}\left(\nu_{j}\right) \mathrm{c}^{4}}
\end{aligned}
$$

Here $N$ is the number of mother nuclei, $G_{f}$ the universal Fermi coupling constant, $\Theta_{c}$ the Cabibbo angle, $M$ the nuclear decay matrix element, $F(E, \mathrm{Z}+1)$ the Fermi function, $p$ the electron momentum, $m_{e}$ the electron mass and $E_{0}$ the $Q$ value of the $\mathrm{T}_{2}$ decay minus the recoil energy of the daughter. $E_{0}$ marks the endpoint of the $\beta$ spectrum in case of zero neutrino mass. For the decay of molecular $\mathrm{T}_{2}$ to the groundstate of the daughter molecular ion $\left({ }^{3} \mathrm{HeT}\right)^{+}$ one derives from the most precise direct determination of the mass difference $m(\mathrm{~T})-m\left({ }^{3} \mathrm{He}\right)=(18590.1 \pm 1.7) \mathrm{eV} / \mathrm{c}^{2}$ [30] an endpoint energy of $E_{0}=(18574.3 \pm 1.7) \mathrm{eV}$ [24] by taking into account the effects through recoil energy and apparative effects ${ }^{1}$. This is in good agreement with [25].

With respect to the required energy resolution, this rather low endpoint favours the choice of tritium. Moreover, the minimal number of electrons in the daughter molecule facilitates the precise calculation of its excitation spectrum $\left(P_{i}, V_{i}\right)$ in $\beta$ decay. Another advantage of tritium decay is its superallowed character with a matrix element as large as $M=5.55$ [17. This leads to a reasonably short half life of 12.3 a and high specific activity of about $3 \mathrm{MBq}$ per $\mathrm{cm}^{2}$ and monolayer from a frozen $\mathrm{T}_{2}$ source, in use here.

The Fermi function can be approximated by 31]

$$
F=\frac{x}{1-\exp (-x)} \cdot\left(1.002037-0.001427 \cdot v_{\beta} / \mathrm{c}\right)
$$

with $\mathrm{x}=2 \pi(\mathrm{Z}+1) \alpha \mathrm{c} / v_{\beta}, \alpha=$ fine structure constant, $v_{\beta}=$ velocity of the $\beta$-particle. Radiative corrections to the $\beta$ spectrum are been applied 32,33. However, they are rather small within our present accuracy limits, they give rise to a shift of $m^{2}\left(\nu_{e}\right)$ of a few percent of our total systematic uncertainty. One may also raise the point whether

1 The apparative effects are a combination of electric potential depression, work functions from substrate and electode system and polarization shift. In the given references 2425$]$ the notation $E_{0}$ represents the difference in the electrostatic potential of the point the electron starts on the source and the point it crosses the analyzing plane, which we will later describe as $e U_{0}$ (see Table 1). 
contributions from right handed currents might lead to measurable spectral anomalies [34. We have checked that the present limits on the corresponding right handed boson mass 35 rule out a sizeable contribution within our present experimental accuracy [36]. The excitation spectrum $\left(P_{i}, V_{i}\right)$ of the daughter $\left({ }^{3} \mathrm{HeT}\right)^{+}$has first been calculated by Kolos, followed by a number of refined numerical calculations, e.g. 37. We are using here the most recent ones by Saenz et al. 38. The excitation spectrum is shown in Fig. 2 The first group concerns rotational and vibrational excitation of $\left({ }^{3} \mathrm{HeT}\right)^{+}$in its electronic ground state; it comprises a fraction of $P_{\mathrm{g}}=57.4 \%$ of the total rate. Its mean excitation energy is $1.73 \mathrm{eV}$ for a $\beta$ energy close to the endpoint. The same amount of recoil energy goes into the center of mass motion of the molecule and is considered already in the $E_{0}$ value given above. In solid $\mathrm{T}_{2}$ the recoil may excite some phonons in addition. But in sudden approximation, which is quite valid here, the mean overall recoil energy will even then - for a $\beta$ energy close to the endpoint - remain at $3.76 \mathrm{eV}$, which is the ballistic energy the decaying nucleus would receive in classical mechanics.

After this first so-called elastic group we observe an important gap in the spectrum up to the first excited electronic state of $\left({ }^{3} \mathrm{HeT}\right)^{+}$at $24 \mathrm{eV}$. This gap could in principle be filled by a ${ }^{3} \mathrm{He}+\mathrm{T}^{+}$continuum which starts at the dissociation energy of $4 \mathrm{eV}$. But dissociation on the cost of the $\beta$ - energy is strongly disfavoured in the Born Oppenheimer approximation. At $30 \mathrm{eV}$ the first electronic continuum opens up in which we observe still strong resonances until complete ionisation is achieved in the second continuum beyond $80 \mathrm{eV}$.

In solid $\mathrm{T}_{2}$ the sudden appearance of an additional nuclear charge may also excite a neighbouring molecule. Kolos et al. 39] have calculated the chance of this spectator excitation to be approximately $5.9 \%$ which is taken into account with some modification (see also section 5.6).

\subsection{Sensitivity of the $\beta$-spectrum to $\boldsymbol{m}^{2}\left(\nu_{e}\right)$}

The last 2 terms in (2) are the total energy $E_{\nu}$ and the momentum $p_{\nu}$ of the neutrino. They represent the neutrino phase space and give rise to the parabolic increase of the $\beta$ spectrum below $E_{0}$ for vanishing neutrino mass, shown in Fig. [1 by the dotted and dashed line. The solid line shows the effect of degenerate neutrino masses $m\left(\nu_{j}\right)=m\left(\nu_{e}\right)=$ $10 \mathrm{eV} / \mathrm{c}^{2}$. In case of the dashed and the solid line only the decay into the electronic ground state of the daughter is considered. For $m\left(\nu_{e}\right)=10 \mathrm{eV} / \mathrm{c}^{2}$ the missing decay rate in the last $10 \mathrm{eV}$ below $E_{0}$ is a fraction of $2 \cdot 10^{-10}$ of the total decay rate, scaling as $m^{3}\left(\nu_{e}\right)$.

We learn from these numbers that the tiny useful high energy end of the spectrum is threatened by an enormous majority at lower energies. However, it can be rejected safely by an electrostatic filter which can be passed only by electrons with a kinetic energy $E$ larger than a potential barrier $q U$ to be climbed. Any momentum analyzing, e.g. magnetic spectrometer cannot guarantee this strict rejection since scattering events may introduce tails to both sides of the resolution function.

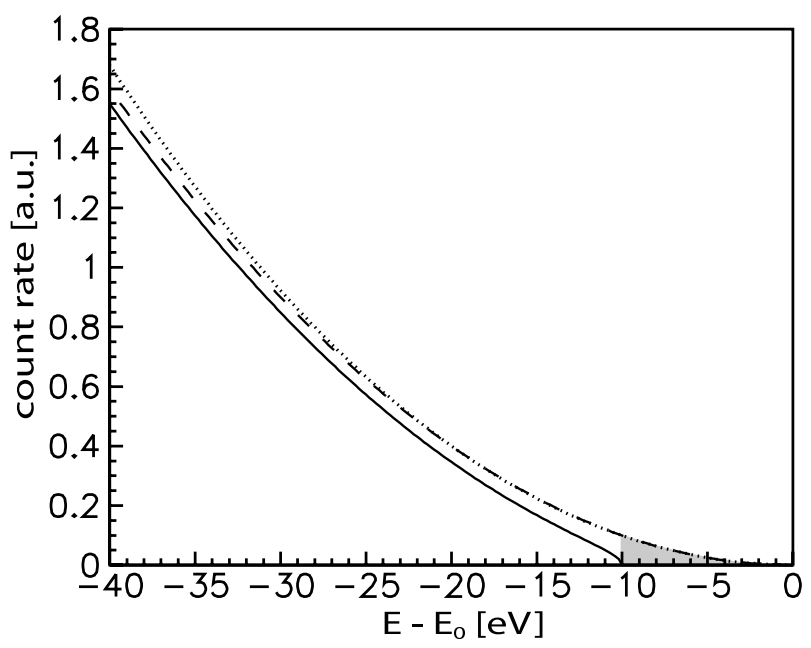

Fig. 1. Tritium $\beta$ spectrum close to the endpoint $E_{0}$. The dotted and the dashed line correspond to $m\left(\nu_{e}\right)=0$, the solid one to $m\left(\nu_{e}\right)=10 \mathrm{eV} / \mathrm{c}^{2}$. In case of the dashed and the solid line only the decay into the electronic ground state of the daughter is considered. For $m\left(\nu_{e}\right)=10 \mathrm{eV} / \mathrm{c}^{2}$ the missing decay rate in the last $10 \mathrm{eV}$ below $E_{0}$ (shaded region) is a fraction of $2 \cdot 10^{-10}$ of the total decay rate, scaling as $m^{3}\left(\nu_{e}\right)$.

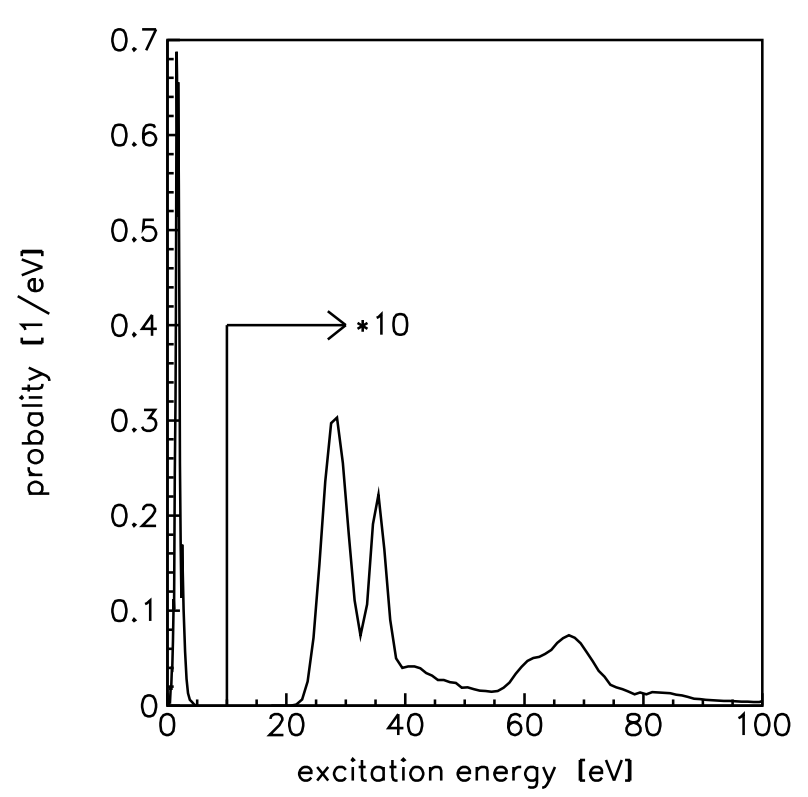

Fig. 2. Excitation spectrum of the daugther $\left({ }^{3} \mathrm{HeT}\right)^{+}$in $\beta$ decay of molecular tritium 38].

Actually, the $m\left(\nu_{e}\right)$ relevant signature of the spectrum extends further below the shaded triangle of missing count rate in (Fig. 1) into the region where $m\left(\nu_{e}\right)$ causes an asymptotically constant offset. Let us investigate this for a sharp filter which integrates the spectrum for energies $E>|q U|$. For short intervals we may treat all factors in front of the sum in (2) as constant. In this interval it is sufficient to consider only the dominant decay mode into the electronic ground state (Fig. 1). We then obtain the 
integral count rate

$$
\begin{aligned}
R(E) & =\sum_{j} \int_{E}^{E_{0}-m\left(\nu_{j}\right) \mathrm{c}^{2}} \frac{\mathrm{d} R}{\mathrm{~d} E^{\prime}} \mathrm{d} E^{\prime} \\
= & C_{R} \sum_{j}\left|U_{e j}\right|^{2}\left(\left(E_{0}-E\right)^{2}-m^{2}\left(\nu_{j}\right) \mathrm{c}^{4}\right)^{\frac{3}{2}}+b \\
& =S+b
\end{aligned}
$$

where $b$ is the background rate, supposed to be independent of the filter setting and $C_{R}$ is a specific signal rate. Under practical conditions the signal rate $S$ integrated over the measurement time $t$ separates from the background noise $\sqrt{b t}$ only at distances $E_{0}-E$ considerably larger than the sensitivity limit on the mass. There we may develop (4) to first order

$R(E)=C_{R}\left(\left(E_{0}-E\right)^{3}-\frac{3}{2}\left(E_{0}-E\right) \sum_{j}\left|U_{e j}\right|^{2} m^{2}\left(\nu_{j}\right) \mathrm{c}^{4}\right)+b$.

Besides the leading cubic term this approximate integral spectrum displays a product of the interval length $\left(E_{0}-E\right)$ and a weighted squared mass

$$
m^{2}\left(\nu_{e}\right)=\sum_{j}\left|U_{e j}\right|^{2} m^{2}\left(\nu_{j}\right)
$$

which is our observable. Hence we call the square root of (6) the electron antineutrino mass $m\left(\nu_{e}\right)$ (see also [20]).

The statistical noise $\sqrt{N}$ on the number of counts $N=$ $(S+b) t$ after a measuring time $t$ will be dominated near $E_{0}$ by the background and further below by the cubic term. The noise of the latter rises like $\left(E_{0}-E\right)^{\frac{3}{2}}$ and hence faster than the mass dependent signal. In between there must be a point with optimal sensitivity on $m^{2}\left(\nu_{e}\right)$; it is found at

$$
S=2 b .
$$

From a measurement at that point for a time $t$ one would calculate [40] a statistical uncertainty by the help of (5)

$$
\delta m^{2}\left(\nu_{e}\right) \mathrm{c}^{4}=\left(\frac{16}{27}\right)^{1 / 6} C_{R}^{\frac{2}{3}} b^{\frac{1}{6}} t^{-\frac{1}{2}} .
$$

We see that the dependence on the background rate is fortunately much weaker than that on the specific signal rate. For the characteristic parameters of our experiment $C_{R}=1.1 \cdot 10^{-5} / \mathrm{eV}^{3} \mathrm{~s}, b=0.015 / \mathrm{s}$, one finds the optimal point at $14 \mathrm{eV}$ below $E_{0}$ and for the value of (8)

$$
\delta m^{2}\left(\nu_{e}\right) \mathrm{c}^{4}=920(t / \mathrm{s})^{-\frac{1}{2}} \mathrm{eV}^{2} .
$$

Within 10 days measuring time (9) drops to $1 \mathrm{eV}^{2}$. In an actual experiment one needs of course quite a number of measuring points within a reasonable interval in order to fix also the other parameters $C_{R}, E_{0}, b$ and to check the spectral shape in general by a $\chi^{2}$ fit.

At a particular measuring point $E$, an endpoint uncertainty $\delta E_{0}$ correlates to $\delta m\left(\nu_{e}\right)^{2}$ according to (5) as

$$
\delta m^{2}\left(\nu_{e}\right)=\frac{\left(\partial R / \partial E_{0}\right)}{\left(\partial R / \partial m^{2}\left(\nu_{e}\right)\right)} \delta E_{0}=2\left(E_{0}-E\right) \delta E_{0} / \mathrm{c}^{4} .
$$

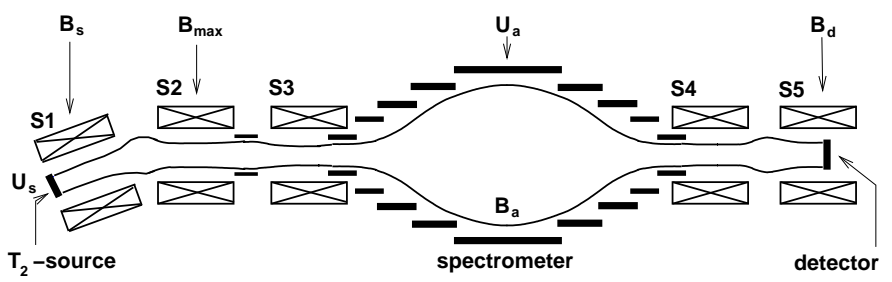

Fig. 3. The improved Mainz MAC-E-Filter is shown schematically. The distance between source and detector is about $6 \mathrm{~m}$ and the diameter of the spectrometer vessel is $1 \mathrm{~m}$. From left to right: Frozen $\mathrm{T}_{2}$ source housed in the tilted solenoid $\mathrm{S} 1$; guiding solenoids S2, S3; the vessel with altogether 27 electrodes; refocussing solenoid S4, S5 housing the detector. The shown magnetic field lines confine the flux tube within which the $\beta$ particles are guided.

Hence $\delta m^{2}\left(\nu_{e}\right)$ increases in proportion to the distance from the endpoint, i.e. the neutrino energy $E_{\nu}$. This is the crux of any missing mass experiment in relativistic kinematics where (10) follows quite generally from the quadratic mass energy relation $m^{2} c^{4}=E^{2}-p^{2} c^{2}$. That underlines again the necessity of measuring the neutrino mass close to the $\beta$ endpoint and disfavours any other experimental concept involving energetic neutrinos in order to gain phase space, i.e. rate.

Instead of fitting $E_{0}$ together with the other parameters from the data one could consider to use the known $Q$ value instead 30 . Its error of $1.7 \mathrm{eV}$, however, would cause through (10) in the most sensitive region, i.e. around $14 \mathrm{eV}$ below the endpoint, an error in $m^{2}\left(\nu_{e}\right)$ of about $50 \mathrm{eV}^{2} / \mathrm{c}^{4}$. This is far beyond our present value obtained from an inclusive fit. The latter is sensitive only to the easily measured small voltage differences in the scan rather than to the absolute energy scale.

On the other hand, we learn from (10) that $E_{0}$ should be fitted including somewhat larger distances from $E_{0}$, since its uncertainty $\delta E_{0}$ decorrelates from $\delta m^{2}\left(\nu_{e}\right)$ like $1 /\left(E_{0}-E\right)$. Altogether, there are in principle three spectral regions from which the basic parameters: $b, m^{2}\left(\nu_{e}\right), E_{0}$ are fitted most sensitively and with a minimum of crosstalk:

(i) a region beyond $E_{0}$ fixing $b$,

(ii) a region shortly below $E_{0}$ fixing $m^{2}\left(\nu_{e}\right)$ and

(iii) a region further below $E_{0}$ fixing $E_{0}$.

In the latter region, however, the inelastic components of the spectrum and their uncertainties start to matter which finally dominate the systematic error. Hence we expect an optimal length of the measuring interval at which we meet a proper balance between the systematic und statistical uncertainty of the result.

\section{Improvements of the Mainz MAC-E-Filter}

$\beta$-spectroscopy in the endpoint region by an electrostatic filter is particularly advantageous in combination with an electron optics based on the principle of magnetic adiabatic collimation (MAC-E-Filter) [21,22]. Particles of charge $q$ are transported from the source to the detector by spiraling along the lines of a magnetic field $B$ connecting 
both (Fig. 31). Hence they can be accepted in the full forward solid angle of $2 \pi$, in principle. An electrostatic filter potential $U$ in between is passed if the longitudinal energy $E_{\|}$along the guiding $B$-line is larger than $q U$. In order to filter the full energy sharply the particle momenta have to be well collimated along $B$. This is achieved by lowering the field strength from a very high value $B_{\max }$ to a quite small one $B_{\mathrm{a}}$ in the region of the analyzing potential. Thereby the transverse energy in the cyclotron motion $E_{\perp}$ is reduced adiabatically in proportion to the magnetic field strength and transformed into longitudinal energy $E_{\|}$along $B$. The non relativistic limit the transformation reads:

$$
\frac{E_{\perp \mathrm{a}}}{E_{\perp \max }}=\frac{B_{\mathrm{a}}}{B_{\max }}=\frac{\Delta U}{U} .
$$

(11) defines the relative width $\Delta U / U$ of a MAC-E-Filter. If the field maximum is placed at the source, $B_{\max }=B_{\mathrm{s}}$, then it accepts the full forward solid angle $\Delta \Omega=2 \pi$.

In reality we have limited the acceptance to a maximum start angle $\Theta_{\max }$ by placing a field maximum $B_{\max }>$ $B_{\mathrm{s}}$ in between source and analyzing plane acting as magnetic mirror for particles starting at angles $\Theta>\Theta_{\max }$ with

$$
\Theta_{\max }=\arcsin \sqrt{B_{\mathrm{s}} / B_{\max }}
$$

Moreover, the angular distribution is slightly modified from isotropy by a scanning potential $U_{\mathrm{s}}$ on the source. Still the transmission function is analytic 41. For charges $q=-e$ it is given in the 4 adjacent intervals

$$
\begin{gathered}
(i): E-e U_{\mathrm{s}} \leq-e U_{\mathrm{a}} \\
(i i):-e U_{\mathrm{a}}<E-e U_{\mathrm{s}}<-e \cdot U_{\mathrm{a}} B_{\max } /\left(B_{\max }-B_{\mathrm{a}}\right) \\
(\text { iii }):-e U_{\mathrm{a}} B_{\max } /\left(B_{\max }-B_{\mathrm{a}}\right) \leq E-e U_{\mathrm{s}} \leq E B_{\max } / B_{\mathrm{s}} \\
(i v):\left(E-e U_{\mathrm{s}}\right) / E \geq B_{\max } / B_{\mathrm{s}}
\end{gathered}
$$

by

$$
T=\left\{\begin{array}{l}
0 \\
1-\sqrt{1-\frac{E-e U_{\mathrm{s}}+e U_{\mathrm{a}}}{E}} \frac{B_{\mathrm{s}}}{B_{\mathrm{a}}} \\
1-\sqrt{1-\frac{E-e U_{\mathrm{s}}}{E} \frac{B_{\mathrm{s}}}{B_{\mathrm{a}}}} \\
1
\end{array}\right.
$$

The second line of (13) describes the sharp rise of the transmission from 0 to a plateau within the filter width $\Delta U_{\mathrm{s}}=\Delta U$. The third line describes a further, slow rise of the transmission in the plateau region as function of an accelerating, thus forward focussing scanning potential $U_{\mathrm{s}}$ on the source until the mirror function of $B_{\max }$ is ruled out in $(i v)$. Transmitted electrons are refocussed by solenoid $\mathrm{S} 4$ and hit a silicon detector in the centre of another solenoid $\mathrm{S} 5$ at a reduced field strength $B_{\mathrm{d}}=0.31 \cdot B_{\max }$. This limits the angle of incidence to $34^{\circ}$. By the help of auxiliary coils around the central part of the spectrometer,
$B_{\mathrm{a}}$ can be varied independently and hence the resolution through (11] 13). A ratio

$$
B_{\mathrm{a}} / B_{\mathrm{s}}>A_{\mathrm{s}} / A_{\mathrm{a}}
$$

has to be observed, however, in order to keep the cross section of the beam carrying flux in the analyzing plane well inside the cross section $A_{\mathrm{a}}$ of the cylindrical electrodes. $A_{\mathrm{s}}=2 \mathrm{~cm}^{2}$ is the cross section of the $\mathrm{T}_{2}$ source. We have been running at field ratios down to $B_{\mathrm{a}} / B_{\mathrm{s}}=3.3 \cdot 10^{-4}$, which limits the flux tube diameter to $88 \mathrm{~cm}$ as compared to the diameter of $94 \mathrm{~cm}$ of the central electrode.

These relations play a role for the background since the electrodes will emit secondary electrons when they are hit by cosmic rays or any other particles originating from radioactivity. If accelerated toward the detector these electrons will arrive with an energy close to that of the transmitted $\beta$-particles and cannot be discriminated by the $1.4 \mathrm{keV}$ (FWHM) resolution of the detector. It is important, therefore, that these secondary electrons are being guided adiabatically along magnetic field lines which pass by the detector. Still we observe enhanced background on its outermost ringsegments. Moreover the central guiding field should not be lowered below $B_{\mathrm{a}} \approx 5 \cdot 10^{-4} \mathrm{~T}$ in order to guarantee full transmission of the $200 \mathrm{eV}$ energy interval under study [40]. Another set of correction coils around the spectrometer annuls the transverse component of the earth's magnetic field and steers the $\beta$ flux. Runs were performed at settings $B_{\max }=2.211 \mathrm{~T}, B_{\mathrm{a}}=5.67 \cdot 10^{-4} \mathrm{~T}$, $B_{\mathrm{s}}=1.087 \mathrm{~T}\left(\Theta_{\max }=44.5^{\circ}\right)$ or $B_{\mathrm{s}}=1.693 \mathrm{~T}\left(\Theta_{\max }=\right.$ $\left.61.6^{\circ}\right), U_{\mathrm{a}}=-18690 \mathrm{~V},-20 \mathrm{~V} \geq U_{\mathrm{s}} \geq-320 \mathrm{~V}$. More details on the general setup and function of the Mainz MAC-EFilter have been given in 21,41, and on its recent improvements and performance in 42,43,44.

\subsection{The new source section}

In the following we will focus on the various improvements of the apparatus, performed in the years 1995-97 42. A decisive improvement concerns the replacement of the LHe bath cryostat by a flow cryostat which allowed to cool down the $\mathrm{T}_{2}$-carrying substrate below $2 \mathrm{~K}$ by a horizontal cooling section, designed and built by Oxford Instruments on customer's demand. Below that temperature the shock condensed, amorphous $\mathrm{T}_{2}$-films have been proven to be stable in time. Earlier the source had been operated at temperatures between $3 \mathrm{~K}$ and $4 \mathrm{~K}$, at which these films turned out to dewet from the substrate and to contract into small crystals with an average thickness much larger than that of the original film 45 46 47]. Within these crystals the chance for multiple inelastic scattering events of $\beta$-particles is enhanced, shifting their energy loss spectrum towards higher losses. Undiscovered, this shift is faking a lower endpoint in the fit which in turn drives $m^{2}\left(\nu_{e}\right)$ through the correlation (10) into the unphysical negative sector.

This effect is the stronger, the more the data interval extends towards lower energies where it takes in more of these multiple scattered particles. This trend was clearly 
seen in our first publication, already, and attributed to a yet unidentified additional energy loss component at 75 $\mathrm{eV}$ [25]. Actually, this number makes sense to the multiple scattering explanation, since the average energy loss per scattering event is $(34.4 \pm 3.0) \mathrm{eV}[4]$ and double scattering prevails in these tiny crystals. Duly later, however, we learned about the dewetting possibility of hydrogen films [4] which was not expected to occur below the triple point. We were thus forced to study this phenomenon also for tritium films, determined the decisive activation energy for surface migration to be $45 \mathrm{~K}$ and concluded from that on a dewetting time constant $\tau_{d} \gg 1$ year at $T<2 \mathrm{~K}$ [4]. The substrate temperature throughout running was $(1.86 \pm 0.01) \mathrm{K}^{2}$. Moreover, the source section was upgraded to house a larger $\left(2 \mathrm{~cm}^{2}\right.$ instead of $\left.1 \mathrm{~cm}^{2}\right)$ and thicker source $(\approx 140$ instead of 30 monolayers of $\mathrm{T}_{2}$ ) in order to cope with the strong gaseous $\mathrm{T}_{2}$ source of the competing experiment at Troitsk [23]. As substrate we have used again highly oriented pyrolytic graphite (HOPG) which combines three advantages:

(i) low backscattering due to the low Z,

(ii) atomic flat surface over wide terraces,

(iii) high purity 50 .

The substrate was glued to the copperhead of the cryostat with the silverloaded, heat conducting glue H20E (supplied by Polytec, D-76337 Waldbrunn). It withstood cryo as well as baking temperatures of $410 \mathrm{~K}$.

Also the source preparation section has been modified: $\mathrm{T}_{2}$ gas was released from a heated titanium pellet and fed through a vacuum baked stainless steel capillary and by help of a mechanical UHV manipulator into a cold $(20 \mathrm{~K})$ radiation shielding tube which surrounded the substrate (Fig. (4). The precooled gas then entered a teflon cup with an inner cross section of $2 \mathrm{~cm}^{2}$ which was pressed against the substrate. A kind of diffusor at the inlet ensured a homogenous molecular flow onto the substrate. The isotopic composition was checked by a quadrupole mass filter. The isotopic $\mathrm{T}$ content of the individual sources varied between $63 \%$ and $84 \%$. It was considerably improved as compared to phase I.

Radiation shield and evaporation cup were provided with quartz windows passed by a He-Ne laser beam which monitored on line the growth of the source film by ellipsometry. For a given polarization status of them incident beam, the status of the outgoing beam depends through Fresnel's formulas on the reflection from both sides of the film and on the interference of the partial waves. Hence the complex refractive indices $\left(n_{\mathrm{f}(\mathrm{s})}+i k_{\mathrm{f}(\mathrm{s})}\right)$ of the film (f) and the substrate (s) enter as well as the film thickness $d$ and the angle of incidence $\phi$. Ellipsometry is performed by help of a polarizer and a $\lambda / 4$ compensator in the incident beam and an analyzer in the outgoing beam ahead of a photocell (Fig. 4). Fixing the easy axis of the compensator to $\gamma=45^{\circ}$ with respect to the plane of incidence one searches for that pair of polarizer angle $(\alpha)$ and analyzer

\footnotetext{
2 This number corresponds to the reading at the cryostat itself, the absolut precision of the temperature is known to $0.1 \mathrm{~K}$.
}

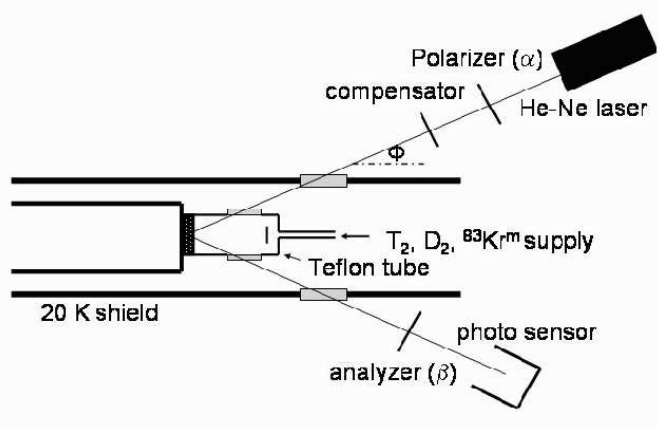

Fig. 4. Scheme of the tritium source with setup for growing the $\mathrm{T}_{2}$ film and controlling its thickness by ellipsometry

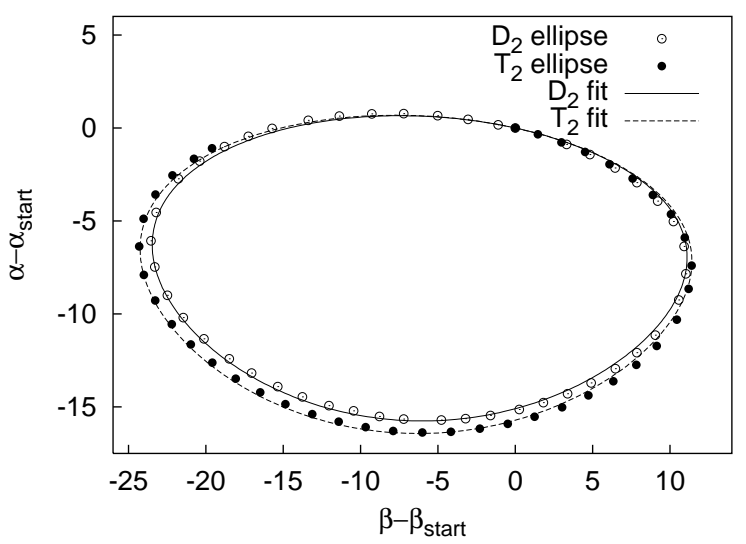

Fig. 5. Control of film growth by ellipsometry for $\mathrm{D}_{2}$ (open circles) and $\mathrm{T}_{2}$ (full circles). On the axes are given the corresponding shifts of light extinguishing $(\alpha, \beta)$ - pairs. The lines are fits to the data. The loop closes at the first interference order.

angle $(\beta)$ for which the reflected beam is extinguished. They are functions of the above parameter set [51].

Except for $k_{\mathrm{f}} \approx 0$ the indices are not known a priori with sufficient accuracy. Hence we have grown stepwise rather thick films of $\mathrm{D}_{2}$ and lately also $\mathrm{T}_{2}$ up to the first interference order at $d=4200 \AA$, determined $(\alpha, \beta)$ pairs of extinction (Fig. (5) and fitted the parameter set to the data with the results (for the example of Fig. [5]) [43]: $\mathrm{D}_{2}$ film: $n_{\mathrm{f}}\left(\mathrm{D}_{2}\right)=1.148, n_{\mathrm{s}}=2.75, k_{\mathrm{s}}=1.34 ; \mathrm{T}_{2}$ film $((65 \pm 10) \% \mathrm{~T},(35 \pm 10) \% \mathrm{H}): n_{\mathrm{f}}\left(\mathrm{T}_{2}\right)=1.156 . \phi$ is measured geometrically to be $(59 \pm 0.3)^{\circ}$; the fit yields about equal value and precision for $\phi$.

The $\mathrm{T}_{2}$ films used in the runs are less than $500 \AA$ thick and the measured $(\alpha, \beta)$ pairs cover only a small section of the full loop, indistinguishable there between $\mathrm{D}_{2}$ and $\mathrm{T}_{2}$. The only parameter safely extracted from this short section is the optical film thickness $n_{\mathrm{f}} d$. For our experiment, however, counts the number column density $\rho_{N} d$, connected to the refractive index by the Clausius Masotti 
relation

$$
\rho_{N}=\frac{n^{2}-1}{n^{2}+2} \frac{3}{4 \pi \alpha}
$$

with $\alpha=0.81(1) \AA^{3}[52$ being the polarizability of hydrogen molecules (for any isotope). From our ellipsometric $n_{\mathrm{f}}$ values we thus determine a molar volume of shockcondensed $\mathrm{D}_{2}: V_{\mathrm{mol}_{2}, \text { shock }}=21.32 \mathrm{~cm}^{3}$ and of

$\mathrm{T}_{2}: V_{\text {mol T}_{2} \text {,shock }}=20.27 \mathrm{~cm}^{3}$ 43. The respective literature values for solid (closely packed) $\mathrm{D}_{2}$ and $\mathrm{T}_{2}$ are $19.95 \mathrm{~cm}^{3} 53$ and $18.9 \mathrm{~cm}^{3} 54$ respectively. The latter is based on calculations. Hence our shock-condensed films exhibit a porosity of

$$
p=\left(1-\frac{V_{\mathrm{mol} \mathrm{c,p}}}{V_{\mathrm{mol}, \text { shock }}}\right)=6.4 \%\left(\mathrm{D}_{2}\right) \text { and } 6.8 \%\left(\mathrm{~T}_{2}\right) .
$$

This is considered in our later discussion on energy loss.

Up to the year 2000, we have analysed the ellipsometry of $\mathrm{T}_{2}$ films using refractive index and molar volume of our shock condensed $\mathrm{D}_{2}$ films. This lead to a systematic underestimation of the $\mathrm{T}_{2}$ column density by $1 \%$. Note, however, that the isotopic composition of our $\mathrm{T}_{2}$ film varies up to $20 \%$ leaving still a slight residual uncertainty about the molar volume of the actual film 43. Moreover, we mention that an alternative calculation of the $\mathrm{T}_{2}$-column density from the measured count rate leads to qualitative agreement but is not sufficiently precise for a quantitative check.

The optical quality of the graphite surface apparently deteriorated somewhat in time, and led to an increase of the relative error of the film thickness from $\pm 3 \%$ (usual case) to $+7 \% /-6 \%$ for the worst case of run Q8 (for more details see 42,43,44]). It is a major contribution to the systematic error through the resulting uncertainty in the energy loss within the source.

After film preparation the source is pushed into the front end of a LHe-cooled chikane, which spans throughout the kinked solenoids S1 und S2. It is the second important improvement of the source section. Evaporating $T_{2}$ is adsorbed on its carbon coating and the straight flight into the spectrometer is prevented by its $20^{\circ}$ bent, whereas the magnetized charged particles follow the equally bent field lines. The cryotrap totally rejected any source dependent background which earlier made up half of the background rate, even for much thinner sources.

The cryotrap also suppressed condensation of rest gas (predominantly $\mathrm{H}_{2}$ ) from the spectrometer onto the $\mathrm{T}_{2}$ film. Still we have observed by an ellipsometric check at the end of a run a certain growth of the film thickness by 0.14 monolayer/day. The source activity, on the other hand, decayed with an apparent lifetime of about one year. Obviously the recoiling daughter molecules each sputtered a handful of neighbouring molecules off the source.

\subsection{Electrode and HV system}

The design of our spectrometer has been aiming at a short, economic instrument with high resolution, that is a high field ratio $B_{\max } / B_{\mathrm{a}}$. Consequently it has sharp $B$ gradients which endanger adiabatic motion. Therefore, we have tried to compensate this drawback by decelerating and reaccelerating the particles partly in the high field within the solenoids S3 and S4. This was provided by a series of ring electrodes.

However, field emission by the strong electric field and particle storage by the strong magnetic field, together favour the development of plasmas even under UHV conditions. Such plasmas lead to an untolerable background rate at field settings $B_{\max } \geq 2 \mathrm{~T}$. Hence the spectrometer could not be operated up to its limit of $B_{\max }=8.6 \mathrm{~T}$ where adiabaticity would be observed best [21].

Therefore, the improvement phase also included a redesign of electrodes No. E6 to E11 in the high $B$ field [42]. Their number was increased by 2 (E12,E13) in order to smoothen the potential drops and titanium was chosen instead of copper for reasons of lower field- and X-ray emission. The latter was suspected to produce through secondary reactions a background component observed about $5 \mathrm{keV}$ above the filter potential whose tail still extends into the accepted energy window of the detector 55 56 , 21]. Moreover, these electrodes were reshaped such that they include about the same magnetic flux $\left(\approx 6 \mathrm{Tcm}^{2}\right)$ everywhere. The new electrode system performed better in so far, as the mentioned background component disappeared, indeed, and a breakdown voltage of $-30 \mathrm{keV}$ could be reached safely within a shorter conditioning phase.

But still the plasma induced background rate rose beyond $B_{\max } \approx 2 \mathrm{~T}$. In the presently running phase III of the experiment, which comprises an extensive background exploration and reduction program to prepare the followup experiment KATRIN [57, we have removed the multielectrode system from the high field region and retained only a few central electrodes, all set to the full analyzing potential. Now the spectrometer is stable up to the highest $B$ field, in accordance with earlier experience at the Troitsk spectrometer. For this latter, non-bakable instrument no stable running mode was found at all with the original multielectrode system [58.

The filter potential $U_{\text {a }}$ was provided by a highly stabilized HV power supply (model HNC5 30000-5 by Knuerr Heinzinger, D-83026 Rosenheim) directly connected to the central electrode. The potentials of the other electrodes (requiring less precision) were derived from $U_{\mathrm{a}}$ by a homemade resistive voltage divider. $U_{\mathrm{a}}$ was monitored and read out continuously by two different systems: The first system comprises a high-precision digital voltmeter (model DMM 6048 by PREMA, D-55129 Mainz) which was connected to $U_{\mathrm{a}}$ via a precision voltage divider 1:5000 (model KV 50 by Julie Research, New York, USA). In the second system the voltage $U_{\text {a }}$ was divided by $1: 50$ by a second voltage divider (model KV 50 by Julie Research, New York, USA) and the difference to a voltage standard (model 335A by Fluke) was measured by a precision digital voltmeter (model DMM 5040 by PREMA, D-55129 Mainz). The observed short- and long-time fluctuations comply with the specifications of the instruments. To check the HV equipment the $\mathrm{K}(32)$ conversion line of ${ }^{83 m} \mathrm{Kr}$ was measured 
Ch. Kraus et al.: Final Results from the Mainz Neutrino Mass Experiment

before or after each tritium run. The values show a small drift from Q2 to Q12 but the difference to the fit values for $U_{0}$ (given in Table 1) of 1998-2001 can be summerized as $749.5 \pm 0.5 \mathrm{eV}$ and appear reasonable compared to the specification of the HV chan. To control the stability during a measurement periode, we analysed shorter time intervals and compared the resulting retarding voltages which are found by the fit for the endpoint values. They agree within their statistical uncertainties.

The scanning potential in the range $-320 \mathrm{~V} \leq U_{\mathrm{s}} \leq$ $20 \mathrm{~V}$ was provided by a fast computer controlled power supply model HNC10 3500-10 by Knuerr Heinzinger, D83026 Rosenheim) and applied to the electrically insulated source. A high-precision divider (Fluke) and a high precision digital voltmeter (model DMM 5017 by Prema, D55129 Mainz). The minimum negative bias of $-20 \mathrm{~V}$ prevents, that recoil ions emmitted from the source are accelerated into the spectrometer where they cause a high background rate through rest gas ionization [25.

\subsection{Spectrometer vacuum and conditioning}

The improvement program also comprised electropolishing of the spectrometer tank and its electrodes, in order to reduce outgassing and field emission but also for removing any tritium contamination from phase I when we were running without the protection by the cryotrap. Also the $80 \mathrm{~m}$ of getter strip (type ST 707/CTAM/30D by SAES, Milano, Italy), mounted onto the inner surface of electrode $\mathrm{E} 2$ were renewed. It represents a pumping speed of $18 \mathrm{~m}^{3} / \mathrm{s}$ for hydrogen. The spectrometer was pumped in addition by 2 turbomolecular pumps at $500 \mathrm{l} / \mathrm{s}$ each.

Once a year the spectrometer was baked for about a week reaching a maximum temperature of $330^{\circ} \mathrm{C}$ to $420^{\circ} \mathrm{C}$ for about 24 hours, at which also the getter was activated. Thereafter the rest gas pressure (mainly $\mathrm{H}_{2}$ ) reached a level of better than $10^{-10}$ mbar. Although we could not observe any deterioration of the vacuum in between, the performance of the spectrometer apparently improved after a very intense rebaking in 2001 in the sense that tiny anomalies appearing in the spectra of runs Q9 and Q10 in 2000, did not occur anymore, thereafter.

In addition to electropolishing and baking, conditioning of the spectrometer up to $\pm 30 \mathrm{kV}$, well above the operating voltage of $18690 \mathrm{~V}$ (neg.), proved to be necessary to prevent any sparkings or minisparkings during runs. The latter are not observed in the electric circuit but manifest themselves by an outburst of background events which die out quite slowly such that a whole scan (passing all measurement points twice) has to be rejected. In the runs of 2001 (Q11,Q12), not a single background burst has been observed. In all earlier runs they appeared about once a week.

\section{$3.4 \beta$-detection and data acquisition}

Transmitted $\beta$-particles are detected by a silicon detector which is segmented into 5 circular rings of $1 \mathrm{~cm}^{2}$ area

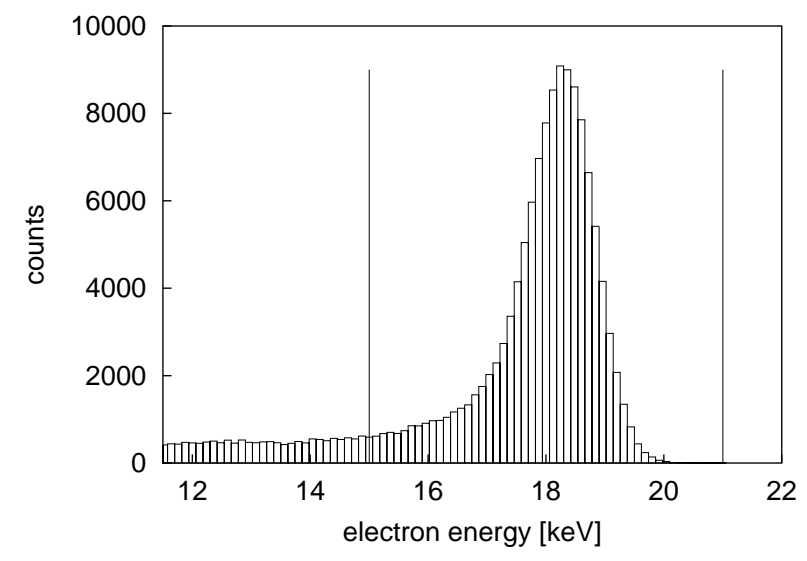

Fig. 6. Detector response to the last $200 \mathrm{eV}$ of tritium $\beta$ decay. The perpendicular lines indicate the accepted window (15$21 \mathrm{keV})$.

each. Usually only the three inner segments are considered for data evaluation, the fourth displays already enhanced background which increases towards the spectrometer walls. The radial segmentation is also useful for accounting for the potential drop which occurs in the centre of the analyzing plane and achieves $4 \cdot 10^{-5} U_{\mathrm{a}}$ on axis.

For phase II the possibly contaminated old detector was replaced by a new one with a still thinner dead layer but otherwise identical specifications (B1256 by Eurisys Mesures, France).The energy loss in the dead layer was determined to be about $200 \mathrm{eV}$ at $E=18 \mathrm{keV}$, corresponding to a mass layer of about $15 \mu \mathrm{g} / \mathrm{cm}^{2}$. The detector together with the attached preamplifiers were cooled down to $\approx-80^{\circ} \mathrm{C}$. The preamplified signals were fed out of the vacuum for further amplification and pulse height analysis. Details are given in [59,60].

Backscattered electrons are remirrored onto the detector within its intrinsic time resolution. They contribute to the low energy tail of the signal with an energy loss by multiple passage through the dead layer. Fig. [6] shows the detector response to the last $200 \mathrm{eV}$ of the tritium $\beta$ spectrum. The FWHM is $1.4 \mathrm{keV}$, the accepted energy window $15 \mathrm{keV}$ to $21 \mathrm{keV}$. Reinforcement of low level lead shielding and removal of some potassium containing material reduced the background from environmental radiation by a factor of 3 down to a rate of $4.6 \cdot 10^{-5} /(\mathrm{s} \mathrm{keV})$ on each segment. Moreover, the vacuum conditions of the detector housing were improved to UHV standards in order to allow removal of the thin foil which earlier had separated it from the much better spectrometer vacuum. It had deteriorated the energy distribution of the passing $\beta$ 's [21]. Instead an open, getter coated tube was installed serving as an active differential pumping section 42.

At fixed energy window of the detector its efficiency slightly increases with $\beta$ energy due to the asymmetric signal slope. By offsetting the $\beta$ spectrum at the source the respective coefficient was determined to be $\alpha_{\mathrm{d}}=(4 \pm$ $2) \% / \mathrm{keV}$. It is considered in the analysis withn marginal effect. The low count rate of less than $250 \mathrm{~Hz}$ allows to acquire the data event by event without suffering substan- 


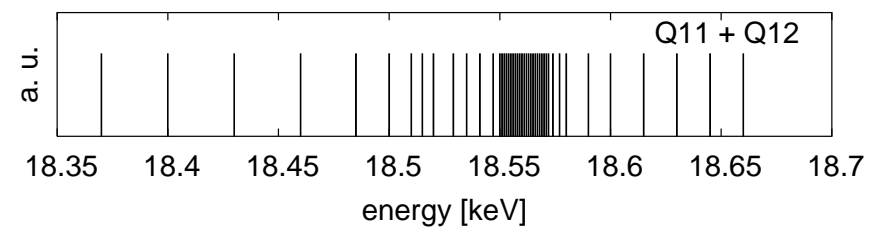

Fig. 7. Distribution of the 45 measurement points in runs Q11 and Q12. In the dense region their spacing is $1 \mathrm{eV}$.

tial losses by signal processing and read out, requiring altogether $63 \mu \mathrm{s}{ }^{3}$. Pile up rejection rises no problem in view of the time constant of $3 \mu \mathrm{s}$ of the analogue circuit. Since any enhanced pile up rate points to electronic noise or some other pertubation it is recorded in order to reject such periods in the off line analysis. The event protocol comprises height and real time of the event. Moreover, its time difference to the foregoing event is recorded with a resolution of $100 \mu$ s for the purpose of correlation studies. Scanning of the spectrum via the source potential $U_{\mathrm{s}}$ is PC controlled. Usually a measuring time of $20 \mathrm{~s}$ per data point was chosen. Their distribution has been adjusted to ensure a properly weighted sensitivity to the decisive fitting parameters. Fig. [7 shows the example of runs Q11 and Q12. Other runs had somewhat different distribution and number of measuring points. The potential differences between data points are ramped with soft slopes over $3 \mathrm{~s}$ in order to prevent particle trapping by sharply rising potential walls. A total scan comprises an up and a down scan. At the end of each data point the filter and source potentials $U_{\mathrm{a}}, U_{\mathrm{s}}$ are read out and stored. Moreover a number of other important control parameters such as the source temperature, the He throughput through the cryostats, the status of the vacuum system etc. are monitored.

Any considerable deviation from normal status activates an automatic control and safety system which communicates the malfunction as short message via mobile phone to the operators in charge. It also performs a safety shutdown if necessary. Vice versa the operators could access the control system at any time and read out the essential parameters remotely. Except for serving hours, therefore, the experiment was running around the clock in a stand alone mode, a necessity in view of the small crew involved.

\section{Measurements in phase II, 1997-2001}

\subsection{Spectrometer background}

Fig. 8] shows a typical background spectrum from the spectrometer as measured by the detector with the $\mathrm{T}_{2}$ source closed off, either mechanically by a valve in the beam line or just with respect to the $\beta$ particles by a filter potential above $E_{0}$. The installation of the bent cryotrap has totally suppressed any source dependent background as

\footnotetext{
${ }^{3}$ From 2000 on this number was decreased to $50 \mu$ s due to a faster computer for data aquisition.
}

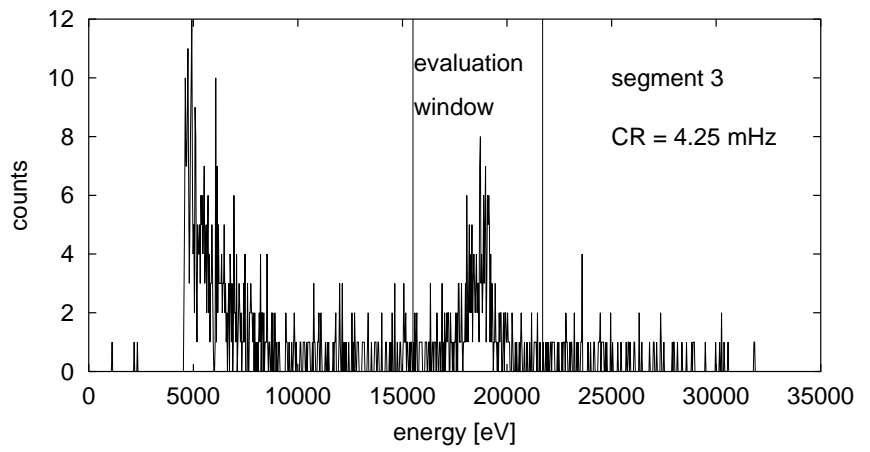

Fig. 8. Spectrometer background spectrum, collected for $13 \mathrm{~h}$ on detector segment 3 . The perpendicular lines indicate the accepted energy window for evaluation.

said before. On top of a smooth continuum one observes a single peak. A high statistics analysis has shown that its mean coincides with the filter potential within an uncertainty of about $30 \mathrm{eV}$. Most probably this peak is a sharp line, actually, stemming from electrons, produced at low energy somewhere in the large analyzing volume of the spectrometer or at the surface of the respective electrodes, and then accelerated by the filter potential towards the detector or the source. According to (11) this is possible, if their transverse energy $E_{\perp \mathrm{a}}$ is less than the filter width of about $4 \mathrm{eV}$. Otherwise they will be trapped magnetically within the $B$ field minimum in the centre.

The rate of this background, which we cannot distinguish from the $\beta$ particles, ranges from about $12 \mathrm{mHz}$ in the very best cases of the last runs Q11 and Q12 up to the order of $50 \mathrm{mHz}$ at poorer performance. The numbers from the similar Troitsk experiment are quite comparable.

The phenomenology of our spectrometer background has been studied extensively in a number of thesises over the years 21,56, 61, 62,63, 64,65, 66. The qualitative insight obtained thereby was very instrumental for improving to the present satisfactory status. But the various mechanisms at work are complex, apparently, such that they could not be identified and disentangled clearly and pinned down quantitatively, neither by experiment nor by simulation. Only the radical hardware measures during the presently running, background dedicated phase III are giving experimental access to a somewhat better understanding of the underlying mechanisms 63, 64. 65.66. Thus routes for further background suppression are opened for KATRIN. Since this will be subject of a forthcoming paper [67, we will confine the background discussion in this paper to the context of phase II runs and results.

From background studies with external $\gamma$ and X-ray sources and from coincidence with passing cosmic muons, it seems to be clear that an important background component - if not all of the observed "hard core" of $12 \mathrm{mHz}$ - consists of secondary electrons emitted from the inner surface of the large central electrodes. In perfect adiabatic motion they would spiral along peripheral flux lines which pass by the detector. However, the actual electromagnetic configuration with its rather weak central $B$ field in com- 

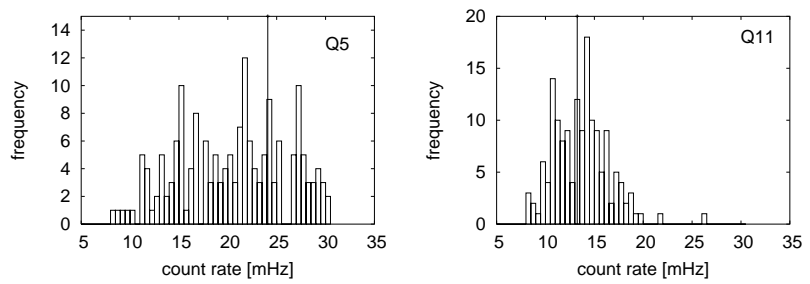

Fig. 9. Histograms of background rates during the measurements Q5 and Q11. Each entry is an average over 8 scans, where the measurement time in the background region adds up to $600 \mathrm{~s}$ for each scan.

bination with radial $E$ field inhomogenities seems to give them a chance to drift into the sensitive flux tube on a non adiabatic path. From muon coincidences we learned that at least part of them arrive within a few $\mu$ s. At UHV conditions, these events cannot be affected any more by rest gas collisions. Recently we have found that such electrons can be rejected by a grid at some repelling potential 63.

Contrary, a single high energy electron, as e. g. from $\mathrm{T}_{2}$ decay within the sensitive flux tube of the spectrometer, may well be stored magnetically for minutes and cause background events by rest gas ionisation with an average rate of the order of the observed one at a vacuum of $10^{-10}$ mbar. At 10 times higher pressure this background source can be recognized (and also eliminated at low signal rate) as a relatively fast chain of correlated events [23. Also minisparks or field emission may end up eventually in such trapped high energy electrons.

Guided by such hypotheses we have applied rf pulses of (1.0-1.8) $\mathrm{MHz}$ and up to $180 \mathrm{~V}$ amplitude onto particular electrodes in order to heat up such trapped electrons stochastically and expel them 27,61. This attempt turned out successful, in fact, when the rf pulse was applied to electrode $\mathrm{E} 8$ on the detectorside which is at a DC potential of $0.86 U_{\mathrm{a}}$. The rf was applied for (1-2) $\mathrm{s}$ each time, during the pause when the scanning voltage was changed. In run Q5 for example we thus managed to reduce the background rate from an unsatisfactory level of $50 \mathrm{mHz}$ down to $\approx 20 \mathrm{mHz}$ [27.

Fig. 9 shows two histograms of background fluctuations, the one from run Q5 with pulsing, the other from run Q11 without pulsing. In the former case pulsing did reduce an enhanced background to an acceptable average rate of $\bar{b}=21.6 \mathrm{mHz}$. But the fluctuation is apparently wider than $\sqrt{b t}$, expected from ordinary statistics. This makes sense, since enhanced background rates during the occasional presence of ionizing trapped electrons obey a kind of Levi statistics with irregular fluctuations. On the other hand, the low and steady "hard core" background of $\bar{b}=12.6 \mathrm{mHz}$, achieved in Q11, displays a nearly ordinary fluctuation.

At UHV conditions, also the build-up and decay of trapped plasmas will occur on longer time scales [21. A certain phase space of electrons can be trapped everywhere within the sensitive flux tube of the spectrometer, either by magnetic mirroring at both necks of the magnetic bot- tle or only at one of them and electrostatic reflection at the central filter potential.

Outside the sensitive flux the stepwise increase of the diameter of electrodes forms small equipotential corners crossed twice by magnetic field lines. This is the electromagnetic configuration of Penning traps. Electrons released from plasmas in such traps may eventually find their way to the detector. Also ions from such plasmas, positive as well as negative ones, can contribute to the backgroud by secondary reactions as said before.

If trapped plasmas are fed at least partly by $\beta$ particles from the source, then the background they produce will depend on the $\beta$-flux therein, which varies with the scanning potential of the source. Such kind of cross talk between scanning and background could be the origin of the tiny residual spectral anomalies which have been observed in few of the runs and reported already in 27. Their amplitude is on the level of $\mathrm{mHz}$, i.e. of order $10 \%$ of the total background rate. The slow build-up and decay rates of stored particles may also give rise to the hysteresis of these anomalies which has been observed between up and down scanning in run Q9.

In a pulsed mode of running, the technique of which has been described in 68, we have searched for a background dependence on the scanning voltage in a time window ranging from a few $\mu$ s to 30 min after shutting off the signal rate by a positive pulse on the source. Within statistical limits of $1 \mathrm{mHz}$ we did not observe any correlation between the background rate in the pauses and the signal rates in the open phases of the spectrometer 62 . This negative result does not really contradict our presumptions on the possible origin of the residual spectral anomalies, since these have only rarely occured at non optimal conditions.

Total stability of the background rate over the full running period has been observed for runs Q11 and Q12.

\subsection{Discussion of Runs Q1 to Q12}

In Table 1 we have listed characteristic parameters of all 12 runs Q1 to Q12 performed with the improved setup in the period 1997 to 2001. They covered an interval from $18370 \mathrm{~V}$ up to $18860 \mathrm{~V}$, i.e. from $200 \mathrm{eV}$ below to $90 \mathrm{eV}$ above $E_{0}$ (Fig. (7).

We should mention that we fitted the parameter $m^{2}\left(\nu_{e}\right)$ as really free parameter without constraints. In order to account for statistical fluctuations of the data the fitting routine ${ }^{4}$ requires a mathematical continuation of the spectrum into the region $m^{2}\left(\nu_{e}\right)<0$ which provides a symmetric $\chi^{2}$ parabola around $m^{2}\left(\nu_{e}\right)=0$ for a statistical data sample. This purpose is fulfilled quite well by introducing

\footnotetext{
4 The shape of the $\beta$ spectrum near the endpoint is mainly defined by the factor $\left(E_{0}-E\right) \sqrt{(Q-E)^{2}-m^{2}\left(\nu_{e}\right) c^{4}}$ which can be expanded for $(Q-E) \gg m\left(\nu_{e}\right) c^{2}$ into $\left(E_{0}-E\right)^{2}-$ $m^{2}\left(\nu_{e}\right) / 2$. Therefore for a neutrino mass around zero any fluctuation of the count rate downwards yields a positive value for $m^{2}\left(\nu_{e}\right)$ and vs. any fluctuation upwards should result in a negative value of the parameter $m^{2}\left(\nu_{e}\right)$.
} 
Table 1. Parameters for measurements Q1-Q12 of phase II. $\Theta_{\max }=$ maximal opening angle, $t=$ running time, $p t=$ number of different measurement points, $f t=$ film thickness, $\bar{b}=$ average background rate, $m^{2}\left(\nu_{e}\right)=$ fit result for the last $70 \mathrm{eV}$ and $U_{0}=$ retarding voltage at which fit finds endpoint value.

\begin{tabular}{|l|l|l|l|l|l|l|l|}
\hline no. & $\Theta_{\text {ma: }} t$ & $p t$ & $\begin{array}{l}f t \\
{[\mathrm{~d}]}\end{array}$ & $b[\mathrm{mHz}]$ & $\begin{array}{l}m^{2}\left(\nu_{e}\right) \\
{\left[\mathrm{eV}^{2}\right]}\end{array}$ & $U_{0}[\mathrm{~V}]$ \\
\hline Q1 & $45^{\circ}$ & 6 & & 20.8 & & \multicolumn{2}{|c|}{ test measurement } \\
\hline Q2 & $45^{\circ}$ & 26 & 50 & 96.7 & $16.7 \pm 0.3$ & $-11.2 \pm 6.0$ & $18573.5 \pm 0.3$ \\
\hline Q3 & $45^{\circ}$ & 24 & 64 & 49.3 & $12.7 \pm 0.2$ & $-14.8 \pm 4.6$ & $18574.0 \pm 0.2$ \\
\hline Q4 & $45^{\circ}$ & 38 & 64 & 49.5 & $11.7 \pm 0.2$ & $-3.9 \pm 4.7$ & $18574.5 \pm 0.2$ \\
\hline Q5 & $45^{\circ}$ & 46 & 64 & 47.5 & $21.6 \pm 0.2$ & $-3.5 \pm 6.0$ & $18574.4 \pm 0.2$ \\
\hline Q6 & $62^{\circ}$ & 38 & 33 & 43.0 & $12.5 \pm 0.2$ & $+0.4 \pm 7.2$ & $18575.7 \pm 0.2$ \\
\hline Q7 & $62^{\circ}$ & 29 & 33 & 43.2 & $14.3 \pm 0.2$ & $-2.4 \pm 4.9$ & $18575.4 \pm 0.2$ \\
\hline Q8 & $62^{\circ}$ & 54 & 39 & 45.5 & $16.5 \pm 0.2$ & $-0.9 \pm 4.8$ & $18576.2 \pm 0.3$ \\
\hline Q9 & $62^{\circ}$ & 56 & 39 & 44.4 & $18.6 \pm 0.3$ & $-10.9 \pm 3.2$ & $18575.1 \pm 0.2$ \\
\hline Q10 & $62^{\circ}$ & 35 & 45 & 45.5 & $16.6 \pm 0.3$ & $-6.1 \pm 4.8$ & $18574.6 \pm 0.2$ \\
\hline Q11 & $45^{\circ}$ & 31 & 45 & 48.2 & $12.6 \pm 0.2$ & $+1.3 \pm 5.8$ & $18576.7 \pm 0.2$ \\
\hline Q12 & $62^{\circ}$ & 19 & 45 & 48.5 & $12.6 \pm 0.2$ & $-1.0 \pm 6.0$ & $18576.6 \pm 0.2$ \\
\hline
\end{tabular}

factors $f_{i}$ to each electronic final state in (2) defined by

$$
\begin{array}{r}
f_{i}=\Theta\left(-m^{2}\left(\nu_{e}\right)\right) \Theta\left(\epsilon_{i}+\mu\right)\left(1+\frac{\mu}{\epsilon_{i}} e^{-\left(1+\epsilon_{i} / \mu\right)}\right) \\
+\Theta\left(m^{2}\left(\nu_{e}\right)\right) \Theta\left(\epsilon_{i}-m\left(\nu_{e}\right) c^{2}\right)
\end{array}
$$

with $\epsilon_{i}=E_{0}-V_{i}-E, \mu=-0.66 m^{2}\left(\nu_{e}\right) \mathrm{c}^{4}$ [25]. For negative $m^{2}\left(\nu_{e}\right)$ they stretch the spectrum smoothly beyond the respective endpoint up to $E_{0}-V_{i}+\mu$. The negative $m^{2}\left(\nu_{e}\right)$ sector might also be fitted by a physical model, namely the $\beta$ spectrum arising from tachyonic neutrinos 69. But this point would come up only in case of an unambigous experimental negative $m^{2}\left(\nu_{e}\right)$ result. Summaryzing, negative values for the fit parameter $m^{2}\left(\nu_{e}\right)$ are not necessarily unphysical but should obtained within statistical limits as result of an unconstraint fit in $50 \%$ of all data sets if the neutrino mass is around zero.

In the following we will report on each of the 12 runs performed in phase II irrespective of wether it has been selected for the final data set. Thus we take the chance to discuss on the given example carefully experimental effects which might lead to spectral anomalies and systematic uncertainties if undiscovered.

Q1 and Q2: The first run Q1 was devoted to a short test experiment with a relatively weak $\mathrm{T}_{2}$ source. We observed in particular that the background due to $\mathrm{T}_{2}$ evaporation into the spectrometer and decay therein had disappeared as said above. Encouraged by this success we have produced a very thick source Q2 of $967 \AA$ corresponding to 284 monolayers. At this thickness the rate of $\beta$ particles which leave the source without energy loss is already close to its maximum possible value obtained from an infinitely thick source. Running this high source activity turned out to be smooth and stable without any source dependent background problems.

However, the analysis of the data revealed an average shift of the endpoint by $-3 \mathrm{eV}$. This effect was then systematically investigated by freezing ${ }^{83 m} \mathrm{Kr}$ activity on top of $\mathrm{T}_{2}$ films and measuring precisely the energy of its $17.8 \mathrm{keV}$ conversion line as function of the film thickness. This way we have discovered that the film charges up positively by $21.2 \mathrm{mV}$ per monolayer. The corresponding electric field strength of $62.6 \mathrm{MV} / \mathrm{m}$ is necessary to release the positive charges, left over from $\beta$ decay, from their trapping potential within the $\mathrm{T}_{2}$ lattice. These first experiences with the improved set up have been communicated in [70. A thorough analysis of source charging is given in 71]. The linear increase of the charge up voltage throughout the film has to be folded into the transmission function and results in a broadening in addition to an average shift. A systematic uncertainty in the broadening effect of Gaussian shape with variance $\sigma^{2}$ would yield an uncertainty in $m^{2}\left(\nu_{e}\right)$ by $-2 \cdot \sigma^{2}$ [72]. Therefore, we have reduced the film thickness by a factor of 2 in later runs. Moreover the uncertainty of the energy loss weighs heavier in a thick source than in thinner ones. Still, as compared to phase I results, the analysis of Q2 led to a reduction of the unphysical negative $m^{2}\left(\nu_{e}\right)$ value by an order of magnitude (see Fig. 13 in sec. 5).

Q3 and Q4: One might have expected that the residual small negative $m^{2}\left(\nu_{e}\right)$ of order $-10 \mathrm{eV}^{2} / \mathrm{c}^{4}$ still observed in run Q2 would disappear with thinner sources. However, the following runs Q3 and Q4 showed the same problem at similar size but with somewhat different dependence from the fit interval. Moreover, the clearly enhanced $\chi^{2}$ values obtained in fitting Q2, Q3 and in particular Q4 pointed to some residual spectral anomalies in the data (see Fig. 13). The Troitsk group had already reported on a step like anomaly which appeared in their integral spectra with an amplitude of order $10^{-10}$ of the total decay rate and at variable positions in the range from 5 to $15 \mathrm{eV}$ below the endpoint. The change in time of the positions of these steps seemed to be compatible with a half year period, even [28. If attributed to a general physics phenomenon, e.g. a monochromatic line in the $\beta$-spectrum of $\mathrm{T}_{2}$, it should appear in our spectra equally. Fitting such a step into the Q4 spectrum led to a significant reduction of $\chi^{2}$, and lifted $m^{2}\left(\nu_{e}\right)$ to an acceptable value of $\left(-1.8 \pm 5.1_{\text {stat }} \pm 2.0_{\text {sys }}\right) \mathrm{eV} / \mathrm{c}^{2}$, indeed [27]. Also the position at $13 \mathrm{eV}$ below $E_{0}$ and the amplitude of the step of about $6 \mathrm{mHz}$ accorded to the Troitsk picture. In the following runs it was an important issue, therefore, to investigate further and - if possible - eliminate these residual spectral anomalies.

Q5 to Q8: Ahead of run Q5 we found out that an enhanced and fluctuating background rate could be reduced essentially by applying an rf pulse to electrode E8 on the detector side as has been reported above. In particular Q5 profited from this procedure; it reduced the averaged background rate from $50 \mathrm{mHz}$ down to $21.6 \mathrm{mHz}$. Q5 was also the first run whose analysis did not reveal any spectral anomaly any more but yielded an $m^{2}\left(\nu_{e}\right)$ value compatible with zero at good $\chi^{2}$ for any data interval (see Fig 13).

Still the background rate was higher in Q5 than in the foregoing runs which suffered from slight residual anomalies. Apparently these anomalies do not correlate necessarily to a higher average background; but a correlation between background events and the operating cycle of the 
spectrometer - which clearly produces an anomaly - can occur also at low average background. At that point we recall that the anomalous count rate does not exceed a few $\mathrm{mHz}$ and hence constitutes at most a small fraction of the background rate. On the other hand the facts seem to corroborate the assumption that the removal of trapped electrons by pulsing also may brake the correlation between background and operating cycle. Run Q5 was the basis of our result $m^{2}\left(\nu_{e}\right)=\left(-3.7 \pm 5.3_{\text {stat }} \pm 2.1_{\text {sys }}\right) \mathrm{eV}^{2} / \mathrm{c}^{4}$ with the limit $m\left(\nu_{e}\right)<2.8 \mathrm{eV} / \mathrm{c}^{2}$ (95\% C.L.) published in 1999 27. But also the series of data collected in the runs Q3 to Q5 could be analyzed successfully in a so-called "15 eV analysis". Besides the background region above $E_{0}$, only the last $15 \mathrm{eV}$ of the spectrum were considered here together with two more data points further down at $18470 \mathrm{eV}$ and $18500 \mathrm{eV}$ respectively; they were necessary to fix $E_{0}$ with sufficient precision. Thus the "troublesome" region of anomalies was excluded mostly from the fit. The result was $m^{2}\left(\nu_{e}\right)=\left(-0.1 \pm 3.8_{\text {stat }} \pm 1.8_{\text {sys }}\right) \mathrm{eV}^{2} / \mathrm{c}^{4}$, which leads to an upper limit of $m\left(\nu_{e}\right)<2.9 \mathrm{eV} / \mathrm{c}^{2}$ (95\% C.L.) 27.

In between runs Q5 and Q6 the spectrometer was baked again to a maximum temperature of $394^{\circ} \mathrm{C}$ and $\mathrm{HV}$ conditioned. The procedure resulted in a background reduction down to $12.5 \mathrm{mHz}$ observed in Q6. This rate was independent of pulsing as proved by the no pulsing mode which ran alternatively every second day. Q7 was running in the same alternating manner. Without pulsing the background had now increased to $14.7 \mathrm{mHz}$ (the number given in Table 1), whereas it remained essentially stable at $12.7 \mathrm{mHz}$ in the pulsing mode. Permanent pulsing was applied to run Q8, the background had increased further to $16.5 \mathrm{mHz}$. Note that these numbers are averages over the full running period. Without pulsing the background was slowly rising in real time and had to be set back by reconditioning the electrode system a few times during a run.

On the thirty-first day of run Q8 the "apparent" lifetime of the source (as measured from the course of its activity) increased from 300 to 620 days. The event was caused by a sudden coverage of the source with a couple of monolayers of $\mathrm{H}_{2}$, which had been collected before from the residual rest gas onto the shield in front of the source. From there it was released then during a short cooling failure of the shield and partly recollected onto the still cold source. The data from the remaining period yielded a significant negative $m^{2}\left(\nu_{e}\right)$ value, caused by the additional energy loss in the $\mathrm{H}_{2}$ cover. Also under regular conditions the shield could not completely prevent a slow and steady condensation of residual $\mathrm{H}_{2}$ onto the source. A daily coverage by 0.3 monolayers was estimated from ellipsometry (see above) and considered in the analysis causing a small, still significant effect (see below).

The analysis of the data set Q6 to Q8 yielded stable fit results of $m^{2}\left(\nu_{e}\right)$ close to zero at any data interval and with good $\chi^{2}$ (see Fig. 13]). Together with Q5 it improved the result, to:

$$
m^{2}\left(\nu_{e}\right)=\left(-1.6 \pm 2.5_{\text {stat }} \pm 2.1_{\text {sys }}\right) \mathrm{eV}^{2} / \mathrm{c}^{4}
$$

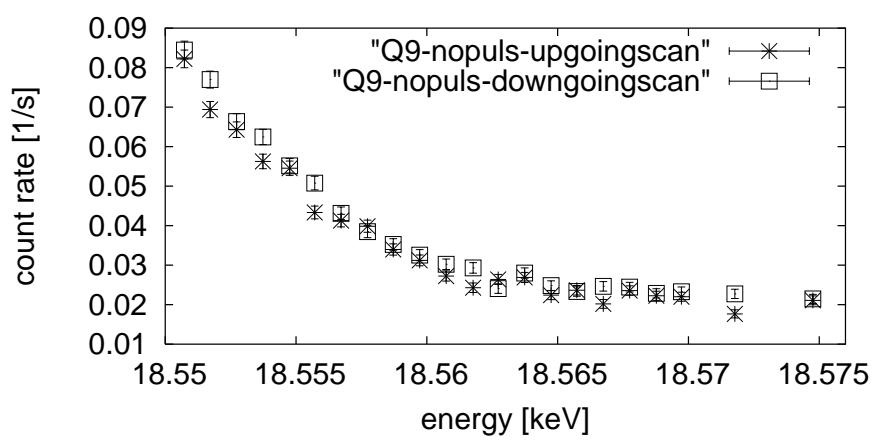

Fig. 10. Hysteresis effect in run Q9 during periods without background suppression by pulsing. Squares correspond to scanning towards the endpoint, crosses to the opposite direction.

from which an upper limit of $m\left(\nu_{e}\right)<2.2 \mathrm{eV} / \mathrm{c}^{2}(95 \%$ C.L.) was extracted. This result has been communicated at the Neutrino 2000 conference [29] and cited thereafter frequently.

Q9 to Q10: The long period of data collection in runs Q5 to Q8 was followed by a number of systematic background studies 6162 the results of which have been summarized above, already. Thereafter tritium measurements were immediately resumed in fall 2000 without a break for extended maintenance. Since the last baking of the spectrometer, which apparently had not reached the temperature of earlier ones, 6 months had elapsed. Although running was quite smooth at a moderate background rate of $20 \mathrm{mHz}$, the on line analysis of Q9 data showed rather soon a reappearance of slightly negative $m^{2}\left(\nu_{e}\right)$ values around $-10 \mathrm{eV}^{2} / \mathrm{c}^{4}$. Nevertheless we continued measuring since parallel runs were foreseen at Troitsk in order to check, whether any Troitsk anomaly would appear synchronously in both experiments. We also refrained from any interference by reconditioning the electrodes like in earlier runs. Rather we decided to watch how the running conditions and results would develop in time in the two alternating modes of cleaning and not cleaning the spectrometer from stored particles by rf pulses.

An apparent anomaly of Q9 is a hysteresis of countrate between up and down scanning: It is visible in the raw spectra already (Fig. 10). Analysis shows that the effect was much stronger in the non-pulsed than in the pulsed mode. The effect also diminished when the final approach to each measuring point was performed always from the same side, namely from a higher voltage level; that means, upramping was performed by first overshooting the proper value and then pulling back as if it were downramping. The hysteresis clearly indicates the presence and influence of stored particles, whose accumulation and/or loss conditions correlate by some mechanism to the setting of the spectrometer. The instability of background conditions introduced this way is also witnessed by an enhanced scatter in Q9 as discussed above and shown in Fig. 9 for the example of Q5. Even if the exact mechanism has not been cleared up yet in detail, the knowledge of its phenomenol- 
ogy is already very important for the experimentalist and enables to prepare cautious countermeasures.

In the following run Q10 the measuring points have not been addressed in a monotonous voltage sequence but in a random generated one. Unfortunately, the software did not allow for a fresh random choice each scan without major changes. But the same sequence was repeated forth and back for the whole run. Still this was sufficient to suppress the conspicious hysteresis of Q9, not so the occurrence of trapped particles as such, however. Hence also the Q10 spectrum is slightly impaired by the imperfect running conditions, leading to a negative $m^{2}\left(\nu_{e}\right)$ result in summary (see Table 1). Q9 and Q10 data have also been analyzed in weekly time bins. They show fluctuations of the $m^{2}\left(\nu_{e}\right)$ fit result which exceed statistical limits. Hence Q9 and Q10 data were not considered in the final fit.

In spite of being slightly impaired, the Q9 and Q10 spectra did not display any indication of a step like Troitsk anomaly, neither in the full data sets, nor in binned ones. This holds in particular for the time bins 6.12.-13.12. and 22.12.-28.12.2000 where the Troitsk experiment was running in parallel to ours. In both periods Troitsk, however, observed the sudden outburst of significant steps. In the second period it even reached an amplitude of $14 \mathrm{mHz}$ (discussed further below).

Q11 and Q12: The experience of Q9 and Q10 has retaught us the importance of optimal maintenance ahead of running, although the vacuum had been fine all the time. Changes in surface conditions seem to rule field emission of electrons and/or ions which fill residual traps and interfere with the measurement. Hence the spectrometer was rebaked at maximum temperature of $373^{\circ} \mathrm{C}$ for $13 \mathrm{~h}$. Thereafter, the electrodes were conditioned up to $\pm 30 \mathrm{keV}$ with reinforced sparking at a residual hydrogen pressure of $10^{-7}$ mbar, obtained by heating up the SAES getters at closed turbo pumps. Also the source section was thoroughly maintained including exchange of source substrate and $\mathrm{T}_{2}$-pellet, baking, etc.

The efforts were rewarded with two absolutely clean and quiet runs Q11 and Q12. From altogether 1620 scans 1580 passed all control criteria in the analysis; among the few rejected runs prevailed incomplete ones due to some peripheral technical problem or intervention. The background rate was stable and further reduced by $20 \%$ to $12 \mathrm{mHz}$ without the necessity of pulsing off stored particles. In Q11 we applied again a random sequence like in Q10, but returned to monotonous scanning in Q12. In the last week of Q12 we ran in a slow scanning mode at $900 \mathrm{~s}$ per point instead of the usual $20 \mathrm{~s}$ interval. This way we searched for possible correlations of rates to scanning steps on an extended time scale but could not identify any.

\section{Analysis of data}

The way we analyze our data has been described before already [25, 27, 29] and will be shortly resumed. In the meantime, we have developed certain refinements, which we also like to apply to the already published data resulting in slight changes of the results.

\subsection{Raw data selection}

The raw data of a run consist of a large number of single event protocols (see above), grouped into single measurements of $20 \mathrm{~s}$ at particular voltage settings. By help of the CERN routine PAW the raw data could be visualized in plots performing cuts of data and correlations of parameters. Outbursts of countrate, e.g. caused by some sparking, were identified and rejected manually this way. In fact the total scan was rejected in case of these rare events since they were followed by a longer "afterglow" of background events. In the latest runs they did not occur at all. Also other obvious malfunctions were identified and rejected this way. This first visual data screening was followed by an automatic one which identified for each single measurement significant deviations of the voltage readings from their nominal value or their average. If they exceeded $0.1 \mathrm{~V}$, the measurement was rejected. This made sure that even at the highest signal rate deep in the spectrum the corresponding signal deviation is less than $0.3 \sigma$. Moreover the program rejected single measurements when they contained more than 10 pile up events. For the remaining data it performed an automatic dead time correction reaching a level of $1 \%$ for the highest count rates. Altogether, the percentage of rejected data ranged from $2 \%$ to $6 \%$ for individual runs.

\subsection{The fit function and the response function}

The data from runs Q2 to Q12 were fitted each by a fit function $F(U)$, which is a convolution of the primary spectrum (2) with the response function $T^{\prime}(E, U)$ of the apparatus plus a constant background $b$ :

$$
F(U)=\int\left(R(E) T^{\prime}(E, U) \mathrm{d} E\right)+b=R \otimes T^{\prime}+b .
$$

$T^{\prime}(E, U)$ is again a fivefold convolution of the transmission function (13), the energy loss function in the film $f_{\text {loss }}$, the charge up potential in the film $f_{\text {charge, }}$ the backscattering function from the substrate $f_{\text {back }}$, and the energy dependence of the detector efficiency $f_{\text {det }}$ :

$$
T^{\prime}(E, U)=T \otimes f_{\text {loss }} \otimes f_{\text {charge }} \otimes f_{\text {back }} \otimes f_{\text {det }} .
$$

It is plotted in Fig. 11 for an electron starting somewhere in the source with energy $E$ and analyzed at a filter setting $U=U_{\mathrm{a}}-U_{\mathrm{s}}$. Its structure is dominated by $T \otimes f_{\text {loss }}$. The filter opens for the elastic component when the retarding potential $-e U$ balances the particle energy, reaching a first plateau $8 \mathrm{eV}$ below. At that point the elastic component is fully transmitted. The slope of $T^{\prime}$ is stretched with respect to that of $T$ by $f_{\text {charge }}$ which spreads over $2.8 \mathrm{eV}$ for a 140 monolayer source. The second, smaller and softer uprise results from integrating up the inelastic spectrum $f_{\text {loss }}$. At a setting of $-e \cdot(U+E)=-50 \mathrm{eV}$ electrons are transmitted to the detector with a chance of $98 \%$ already. $f_{\text {loss }}$ has been determined in parallel for gaseous hydrogen as well as for shock condensed films (actually $\left.\mathrm{D}_{2}\right)$ with the $\beta$ electron spectrometers at Troitsk 


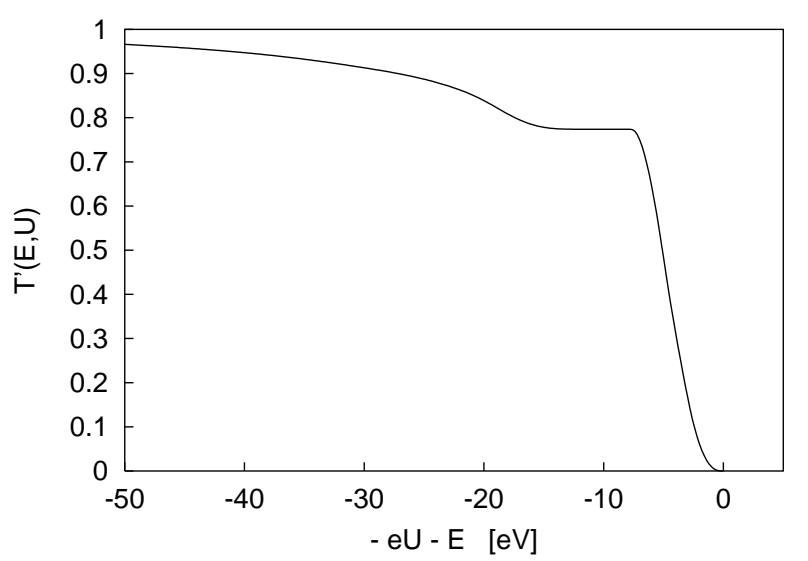

Fig. 11. Convoluted and normalized response function of the apparatus to electrons emitted at energy $E$ and analysed at the filter voltage $U$. The filter width $\Delta U$ is set to $4.8 \mathrm{~V}$, the maximum starting angle to $\Theta_{\max }=45^{\circ}$ and the convolution is calculated for a source thickness of $490 \AA$.

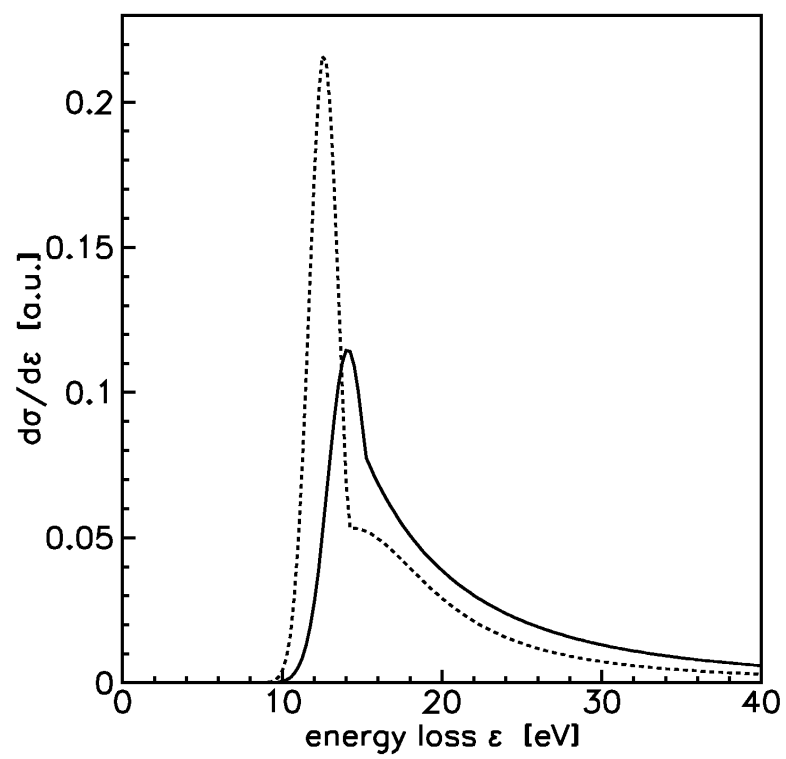

Fig. 12. Differential inelastic cross sections for $18.5 \mathrm{keV}$ electrons scattered from gaseous hydrogen (dashed line) and quench condesend $\mathrm{D}_{2}$ (solid line) [48.

and Mainz, respectively 48. For the condensed case, the differential cross section $\mathrm{d} \sigma / \mathrm{d} E$ was approximated by two model functions, a Gaussian peak at an energy loss of $14.1 \mathrm{eV}$ followed by a third order hyperbola (Fig. 12). The total inelastic cross section was found in this case to be $\sigma_{\text {tot }}=(2.98 \pm 0.16) \cdot 10^{-18} \mathrm{~cm}^{2}$ with a mean energy loss of $(34.4 \pm 3.0) \mathrm{eV}$. As compared to the gaseous phase, it is found that the excitation peak is shifted upwards by $1.5 \mathrm{eV}$ and the total cross section lowered by $13 \%$. The shift is also confirmed by quantum chemical calculations 48. If we define an inelastic scattering coefficient $K$ of the film by

$$
K=\sigma_{t o t} \cdot \rho_{N} \cdot \frac{l}{\cos \Theta}
$$

with $l / \cos \Theta$ being the actual path length of a $\beta$ particle through the film, then the probability of scattering $n$ times is given by a Poisson distribution

$$
P_{\mathrm{ne}}=e^{-K} K^{n} / n !
$$

At the given film thickness it is sufficient to take multiple scattering up to third order into account. The response function is obtained for each tritium film layer at a certain electrical potential - defined by $f_{\text {charge }}$ - by an appropriate convolution of Poisson distributions (22) over energy loss and path length [48. Running at different $\Theta_{\max }$ changes the response function, thus requiring separate evaluation of runs.

Backscattering from the graphite substrate is quite small. Simulations have shown that its spectrum may be approximated within the interesting interval of $200 \mathrm{eV}$ below the starting energy $E$ by a constant pedestal of relative amplitude

$$
\alpha_{\text {back }}=3.1 \cdot 10^{-5} / \mathrm{eV}
$$

with respect to a $\delta$ - function at $E^{\prime}=E$. The latter represents the transmission probability for forward emission [45]. The number given above applies to $\Theta_{\max }=60^{\circ}$. It decreases for a narrower transmission cone of $\Theta_{\max }=$ $50^{\circ}$ to $2.3 \cdot 10^{-5} / \mathrm{eV}$. The simulations have been checked by test experiments with K-conversion electrons from ${ }^{83 m} \mathrm{Kr}$ decay [45].

Since the back scattering effect is small it is sufficient to replace the exact convolution procedure by a simple correction factor

$$
f_{\text {back }, \text { corr }}=1+\alpha_{\text {back }}(E+e U) .
$$

The second term stands for the integral of the backscattered spectrum over the width $(E+e U)$ of the transmission window of the spectrometer. Also the folding with the energy dependent detector efficiency in (20) can be replaced by applying a simple correction factor

$$
f_{\text {det }, \text { corr }}=1+\alpha_{\mathrm{d}}(E+e U)
$$

with the coefficent mentioned before

$$
\alpha_{\mathrm{d}}=(4 \pm 2) \cdot 10^{-5} / \mathrm{eV} .
$$

The fit is then performed with $m^{2}\left(\nu_{e}\right), E_{0}, b$ and a signal amplidtude as free parameters.

\subsection{Fits of individual runs}

For all runs Q2 to Q12 fits were performed on data intervals of different spectral extension. Fig. 13] shows the resulting $m^{2}\left(\nu_{e}\right)$ as function of the lower cut off of the accepted data interval. In some cases we observe small but still significant negative $m^{2}\left(\nu_{e}\right)$ values. For runs Q4 and Q7 the corresponding fit values for $E_{0}$ are shown separately in Fig. 14 Their variation relative to that of $m^{2}\left(\nu_{e}\right)$ reflects grosso modo the correlation (10).

Fig. 13] shows in addition the reduced $\chi^{2}$ values for all fit intervals. In cases of negative or unstable $m^{2}\left(\nu_{e}\right)$ values 


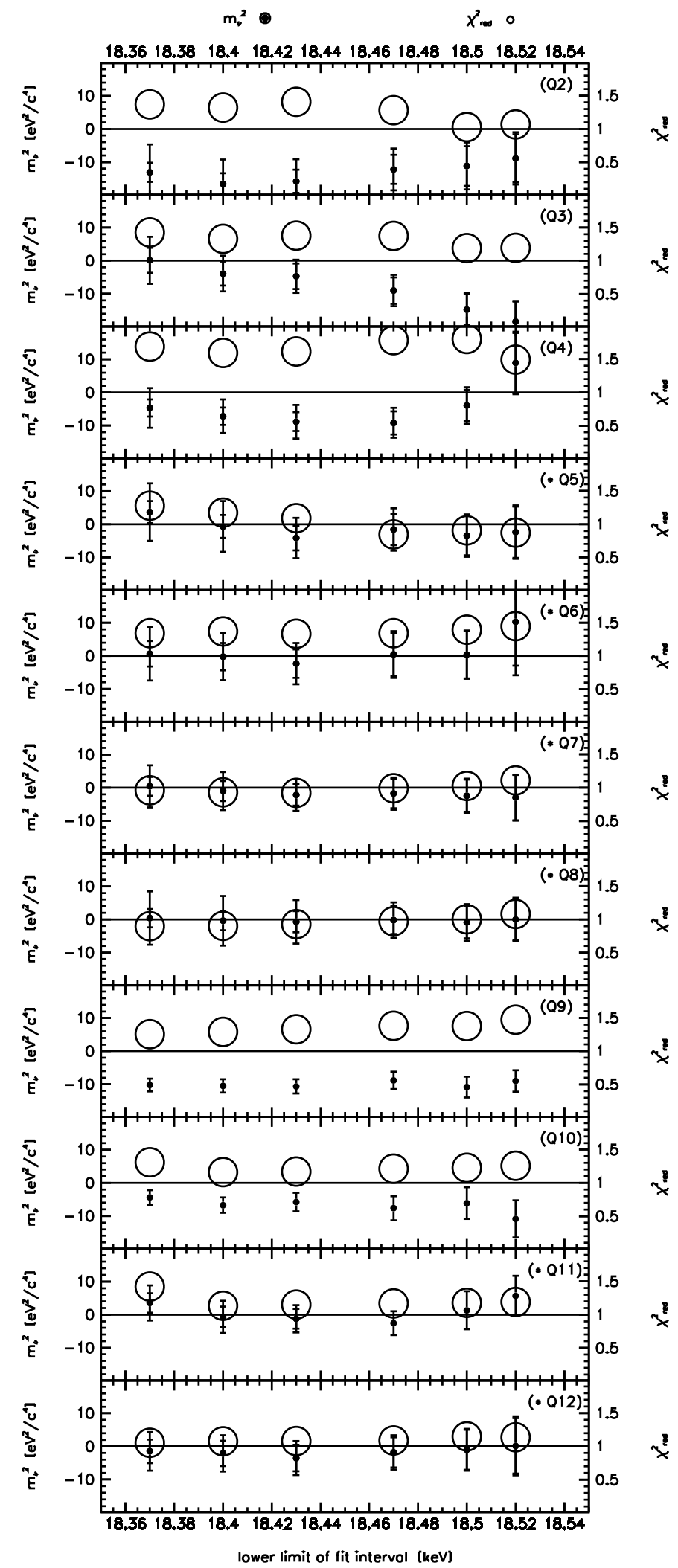

Fig. 13. Fit results of $m^{2}\left(\nu_{e}\right)$ (data points, left scale) and reduced $\chi^{2}$ (circles, right scale) for runs Q2 to Q12 in dependence of the lower limit of the fit interval. The upper limit was always $18.6 \mathrm{keV}$. The inner error bars correspond to the statistical, the outer to the total uncertainty (except for Q9,Q10). The measurements used in the final analysis are marked by a star.

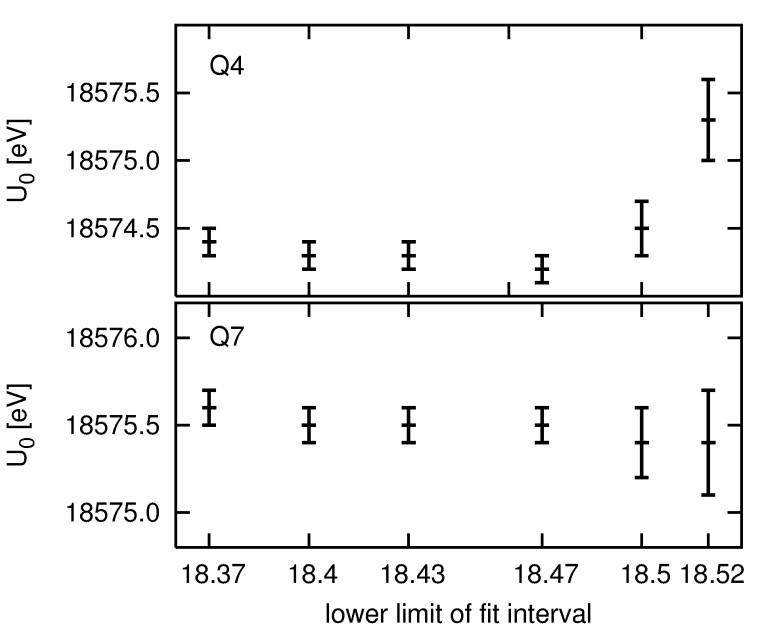

Fig. 14. Retarding voltages at which the fit finds endpoint values for runs Q4 and Q7 in dependence of the fit interval.
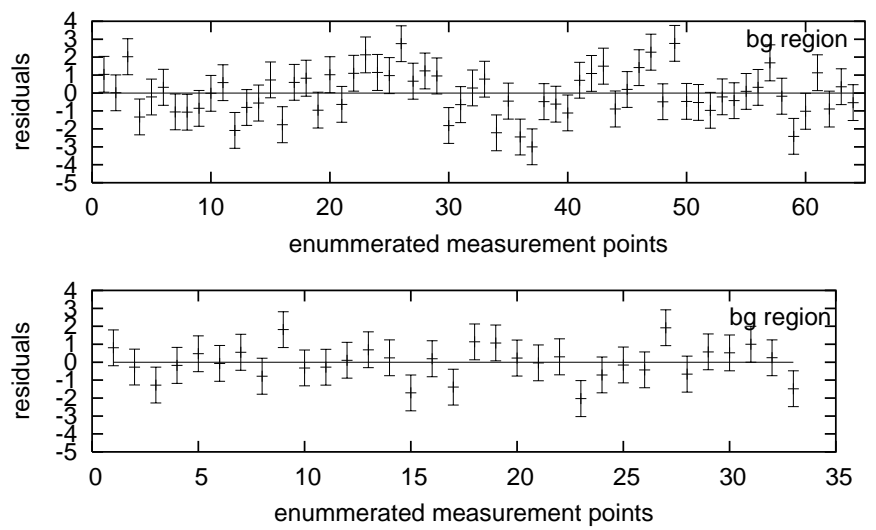

Fig. 15. Residua of fits for runs Q4 (upper part) and Q7 (lower part). The measurement points are enummerated to avoid misleading of the eyes due to unequal distances of the points. From left to right they correspond from $200 \mathrm{eV}$ below to $100 \mathrm{eV}$ above the endpoint $E_{0}$.

they usually exceed 1 , whereas they lie in the optimal range for data sets with straight $m^{2}\left(\nu_{e}\right) \approx 0$ fit results.

$\underset{x}{*}$ This is seen more clearly in Fig. 15 by the comparison of the residua of the straight data set Q7 and the somewhat distorted one Q4.

\subsection{Run selection for the final evaluation}

The high statistics of individual runs in phase II reveals small systematic differences between their results, which have been discussed in the preceding section. Since they fall into two clear-cut classes, clean ones and those with residual problems, we may select only the former ones for the final evaluation in order to minimize systematic deviations and uncertainties and to arrive at our optimum overall result. This procedure might be considered question- 
able if the selection were based on a negative $m^{2}\left(\nu_{e}\right)$ result alone; because it might then introduce a bias. But we have clearly shown that negative and fluctuating $m^{2}\left(\nu_{e}\right)$ values (the latter as function of the data interval) occur at unstable running conditions, in particular with respect to background. Hence we can well justify our choice. In this sense we retain runs Q5 to Q8, Q11 and Q12 for the final analysis. It should be noted, that only starting with run Q5 the very important method of applying high frequency pulses to one electrode at the detector side of the spectromter has been used in order to clean the spectrometer from possibly stored particles and to stabilize the background rate.

In detail Q2 had to be rejected because we had prepared an obviously too thick source from which we learned about the unexpected charge up effect. Here it spread over $6 \mathrm{eV}$, which is probably too much to be corrected for safely. The other 4 rejected runs suffered from small residual spectral anomalies of the data discussed above. Only in run Q4 we observed an anomaly whose signature was compatible with a Troitsk anomaly, i.e. a step rise in the integral spectrum. In this case one might follow the Troitsk procedure of analysis. It consists of fitting 2 additional parameters for position and size of the step to the data. For distinct steps lying not too close to the endpoint the additional parameters decorrelate sufficiently from the mass parameter such that the fit yields reasonably stable $m^{2}\left(\nu_{e}\right)$ values close to zero 28. This is the case for Q4 27] (and actually only for Q4). However, we have decided to retain from this ad hoc procedure, since the step effect is neither stable nor properly understood (see also section 6.2). This way we facilitate at least the discussion of systematic uncertainties below.

\subsection{Joint analysis of selected runs}

The data from the selected runs Q5 to Q8, Q11 and Q12 cannot be simply summed up for a single fit, since they have been collected at somewhat different conditions with respect to source strength, accepted solid angle, choice of measuring points etc. We also have to face slight changes of the fitted endpoint value beyond the statistical limit of order $100 \mathrm{meV}$, since we cannot guarantee the stability of our HV equipment to that level over years. Actually $m^{2}\left(\nu_{e}\right)$ is the only parameter expected to approach one and the same value in any correct data set. Therefore, we have performed a joint fit of the full data set with respect to only this parameter by the following procedure. We have first fitted each of the selected runs separately with respect to amplitude, background, and endpoint, and have calculated its $\chi^{2}$ as a function of the common parameter $m^{2}\left(\nu_{e}\right)$. The six $\chi^{2}$ curves were then added up to form a global $\chi^{2}$ curve (Fig. 16) from which the final $m^{2}\left(\nu_{e}\right)$ fit result and its statistical error are determined. This procedure is equivalent to a common fit of all six data sets with $3 \cdot 6+1=19$ free parameters; but it converges much faster, since it makes proper use of the fact that each subset depends only on three individual and one common parameter.

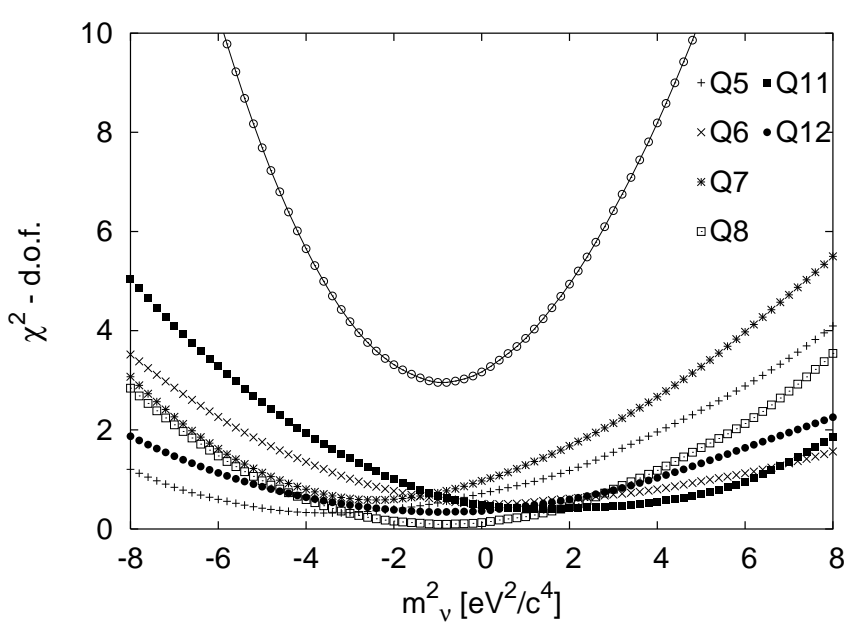

Fig. 16. Determination of $m^{2}\left(\nu_{e}\right)$ from the final data set. Shown are the $\chi^{2}$ plots for the parameter $m^{2}\left(\nu_{e}\right)$ for the single data sets (different symbols) and the sum (open circles), which corresponds to the fit of the total data set. The fit intervals are restricted to a lower limit of $70 \mathrm{eV}$ below $E_{0}$.

\subsection{Uncertainties of input parameters}

For most of the input parameters entering our final fit, we adapt the same values and systematic uncertainties as chosen before in ref.s [27|29]. For the prompt neighbour excitation in solid $\mathrm{T}_{2}$, however, we present in addition a new, critical treatment below. Some uncertainties have been quoted in section 5.2 already. The others are discussed in the following.

Final states of the daughter molecule: We use the most recent calculation by Saenz et al. 38, which have been calculated for gaseous $\mathrm{T}_{2}$ with fully satisfactory precision. In solid $\mathrm{T}_{2}$ the excitation energy of higher excited final states shifts up slightly with respect to the ground state of $\left(\mathrm{T}^{3} \mathrm{He}\right)^{+}$. The effect has been estimated by A. Saenz [73] and is considered here with a correction of $0.8 \mathrm{eV}$ for the second electronically excited state group and with $1.4 \mathrm{eV}$ for the third one. This correction is also considered fully as uncertainty.

Energy loss in the $\mathbf{T}_{2}$ film: Spectrum and cross section of energy loss have been discussed already in section 5.2 . The relative uncertainty of the latter is $5.4 \%$ which is added in quadrature to the one of the column density of individual runs according to Table 1.

Moreover, we consider a continuously growing coverage of the source by 0.3 monolayers of $\mathrm{H}_{2}$ per day. This number has been obtained in two independent ways [43]: (i) Ellipsometric determination of the source thickness at the beginning and end of each run has revealed a growth of the total thickness of 0.14 monolayers per day in the average. Subtracting a $T_{2}$ loss of 0.17 monolayers/day as calculated from the apparent lifetime of the source of 400 days, yields a growing coverage by 0.31 monolayers/day. (ii) Evaluation of data subsets from fresh and older sources shows a significant trend towards negative $m^{2}\left(\nu_{e}\right)$ values for the older ones. A coverage growth of 0.29 monolayers/day raises this dependence. The uncertainty of both 


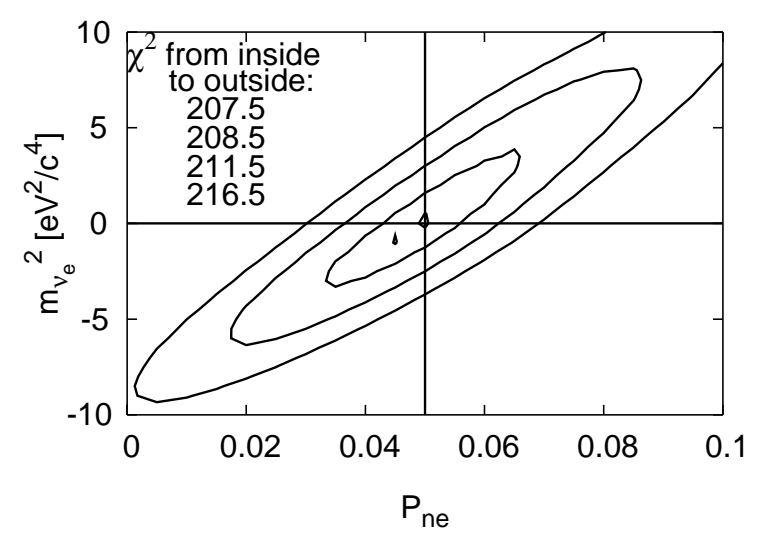

Fig. 17. Shown are contour plots of $\chi^{2}\left(P_{\text {ne }}, m^{2}\left(\nu_{e}\right)\right)$ at $1 \sigma, 2 \sigma$ and $3 \sigma$ around its minimum value at $(0.05,0)$.

results is clearly larger than their difference. Therefore the correction by 0.3 monolayers/day is also considered fully as uncertainty.

Neighbour excitation: The prompt excitation of neighbours next to a decaying $\mathrm{T}_{2}$ molecule has been estimated by Kolos in sudden approximation 39. The effect is due to the local relaxation of the lattice following the sudden appearance of an ion. A rigorous calculation of final states of the surrounding electron cloud is still missing. Therefore, it is difficult to assign a proper uncertainty to Kolos' estimated excitation probability of $P_{\text {ne }}=0.059$ with a mean energy of $14.6 \mathrm{eV}$. The latter number applies to the excitation spectrum of free hydrogen molecules. It seems reasonable to raise this number by the same $1.5 \mathrm{eV}$ by which the energy loss spectrum of electrons is shifted upwards (compare section 5.2). In the same sense, the corresponding reduction of the total inelastic cross section by $13 \%$ 48 has been applied also to $P_{\text {ne }}$ in our former standard analysis. Another reduction of $P_{\text {ne }}$ by $11 \%$ has been accounted for the observed porosity of our shock condensed films (see section 3.1), yielding finally $P_{\text {ne }}=0.046$. This number has also been used in [27, although it has been composed from slightly different factors. Since the shifts in excitation energy and probability, applied to Kolos' calculated values, are based but on qualitative, plausible arguments, they had entered also fully the systematic uncertainty.

The large, consistent data basis available now gives us a handle to try a self-consistent determination of $P_{\text {ne }}$ by treating it as an additional free parameter in a joint analysis of a large interval comprising data down to $170 \mathrm{eV}$ below $E_{0}$ where the inelastic components really matter. Fig. 17 shows the resulting $\chi^{2}$ contour plot in the $\left(P_{\text {ne }}, m^{2}\left(\nu_{e}\right)\right)$ plane. Its minimum lies at $P_{\text {ne }}=0.050 \pm$ 0.016 and $m^{2}\left(\nu_{e}\right)=(0 \pm 3.3) \mathrm{eV}^{2} / \mathrm{c}^{4}$, a very satisfactory

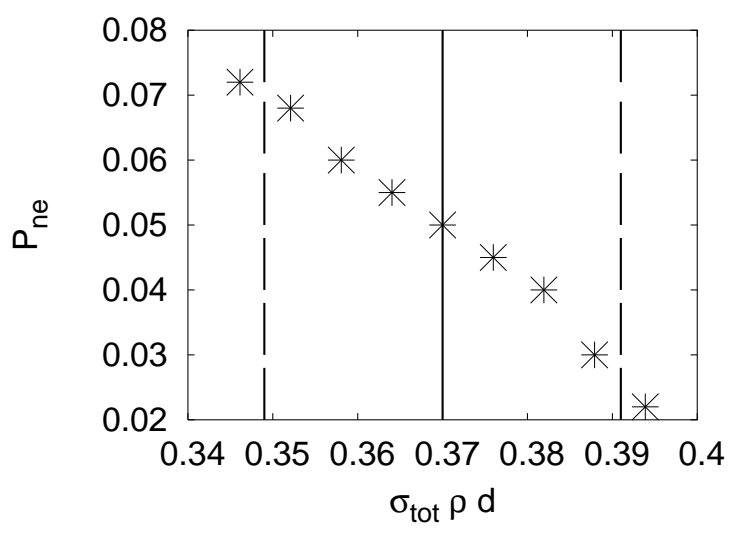

Fig. 18. Correlation between neighbour excitation probability $P_{\text {ne }}$ and $\sigma_{\text {tot }} \rho d$. Each point corresponds to the centre of a contourplot like in Fig. [17 calculated for particular values of $\sigma_{\text {tot }} \rho d$ within its $1 \sigma$ uncertainty interval $(0.370 \pm 0.023)$, indicated by the vertical lines.

result, indeed. It confirms our former, estimated value of $P_{\text {ne }}=0.046$ within errors and lifts the former tendency towards slightly negative $m^{2}\left(\nu_{e}\right)$ values for the entire data set (see below).

Still this fit value of $P_{\text {ne }}$ is subject to a quite sizeable systematic uncertainty of \pm 0.022 . It stems from its strong correlation to the energy loss in the film due to their similar effect on the spectrum. The uncertainty for energy losses are a combination of the uncertainty of the determination of the film thickness and the measurement of the total cross section $\sigma_{\text {tot }}$. The averaged uncertainty in measuring the film thickness for all accepted runs is $3 \%$ and the uncertainty of $\sigma_{\text {tot }}$ is $5.4 \%$. In order to determine the correlation of $P_{\text {ne }}$ and $\sigma_{\text {tot }} \rho d^{5}$ we have calculated the $\chi^{2}$ contour plot of Fig. 17 for different values of $\sigma_{\text {tot }} \rho d$ and located it's minimum in the $\left(P_{\text {ne }}, m_{\nu_{e}}^{2}\right)$ - space each time. The corresponding $\left(P_{\text {ne }}, \sigma_{\text {tot }} \rho d\right)$ - pairs are plotted in Fig. 18 We see an almost straight anticorrelation of the two energy loss contributions. The correlation transfers the uncertainty of $\sigma_{\text {tot }} \rho d$ directly into one of $P_{\text {ne }}$ as indicated by the bars in Fig. 18 $m^{2}\left(\nu_{e}\right)$ and $\chi^{2}$ are rather insensitive to this exchange of $\sigma_{\text {tot }} \rho d$ and $P_{\text {ne }}$. In particular, we cannot fix $\sigma_{\text {tot }} \rho d$ separately by the fit alone better than by external input.

Self-charging of $\mathbf{T}_{2}$ film: It has been found that the film charges up within $30 \mathrm{~min}$ to a constant critical field strength of $62.6 \mathrm{MV} / \mathrm{m}$ [7]. It results in a linearly increasing shift of the starting potential of $\beta$ particles throughout the film, reaching about $2.5 \mathrm{~V}$ at the outer surface. We have assigned a conservative systematic uncertainty of $\pm 20 \%$ to that slope.

Backscattering and detector efficiency: Both effects are small and can be accounted for by the linear correction factors (2324) and (2526) given above. They may just be contracted into a single correction factor

$$
f_{\text {backcorr }} \cdot f_{\text {dcorr }} \approx 1+\left(\alpha_{\text {back }}+\alpha_{\mathrm{d}}\right)(E+e U) .
$$

\footnotetext{
${ }^{5} \sigma_{\text {tot }} \rho d$ is a measure for the scattering probability in the
} tritium film. 


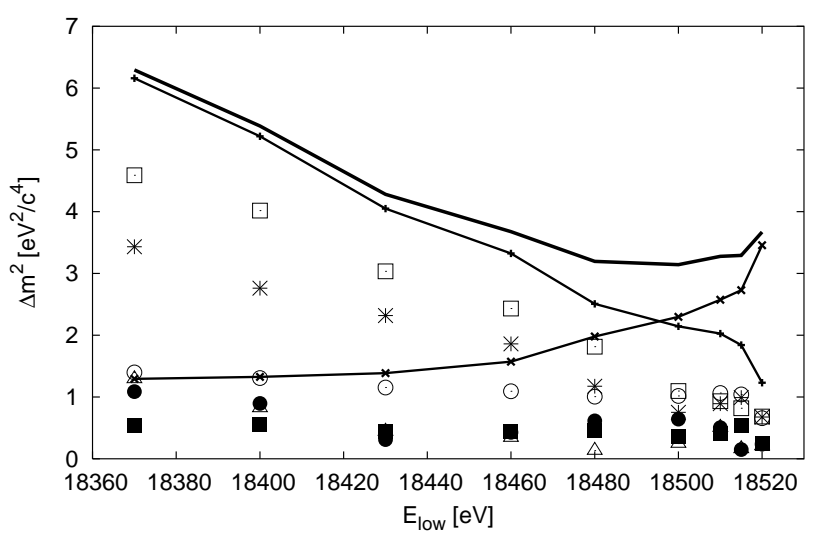

Fig. 19. Individual and quadratically summed up uncertainties of $m^{2}\left(\nu_{e}\right)$ for the joint data set, calculated for different lengths of the data interval. The open squares and stars give the contribution of energy losses in the tritium film, the open circles the neighbour excitation, the filled squares the selfcharging effect, the filled circles the final states and the open triangles the detector efficiency. The line with simple crosses shows the sum of systematic uncertainties in dependence from the fit interval and the line with stars gives the corresponding statistical uncertainty (growing). The upper most line gives the quadratically summed up values and has its minimum for $18500 \mathrm{eV}$.

In (26) we have already assigned to $\alpha_{\mathrm{d}}$ a conservative uncertainty of $\pm 2 \cdot 10^{-5} / \mathrm{eV}$, which is large enough to cover a residual uncertainty of $\alpha_{\text {back }}$ as well.

\subsection{Systematic uncertainty of $m^{2}\left(\nu_{e}\right)$}

In the standard analysis the systematic uncertainty of $m^{2}\left(\nu_{e}\right)$ is calculated from those of the external input parameters as follows: Each input parameter is shifted from its best value by its uncertainty and the fit to a particular data set is repeated. The resulting shift of the $\chi^{2}$ minimum with respect to $m^{2}\left(\nu_{e}\right)$ is then taken as the corresponding systematic uncertainty of $m^{2}\left(\nu_{e}\right)$.

The systematic uncertainty of the joint data set is evaluated in the standard analysis by the same procedure. Fig. 19] shows for the joint data set the individual contributions as well as the quadratically summed up uncertainties calculated for different lengths of the data interval. Obviously the statistical uncertainty decreases with the length of the interval whereas the systematic increases. The total, combined uncertainty attains a flat minimum of $\Delta m_{\mathrm{tot}}^{2}=3.04 \mathrm{eV}^{2} / \mathrm{c}^{4}$ for a lower cut off at $E_{\text {low }}=18500 \mathrm{eV}$ which is regarded the optimum interval, therefore.

Summarizing, the standard analysis with the external input parameter of neighbour excitation probability $P_{\text {ne }}=$ $(0.046 \pm 0.013)$ yields for the optimal data interval the result

$$
\begin{array}{r}
m^{2}\left(\nu_{e}\right)_{\text {standard }}=\left(-1.2 \pm 2.2_{\text {stat }} \pm 2.1_{\text {syst }}\right) \mathrm{eV}^{2} / \mathrm{c}^{4} \\
\chi^{2} / \text { d.o.f. }=208 / 195 .
\end{array}
$$

Here the uncertainties of the externally chosen, independent parameters $P_{\text {ne }}$ and $\sigma_{\text {tot }} \rho d$ contribute to that of $m^{2}\left(\nu_{e}\right)$ by an amount of

$$
\delta m^{2}\left(\nu_{e}\right)_{\text {syst,ext }}\left(P_{\text {ne }}, \sigma_{\text {tot }} \rho d\right)=1.59 \mathrm{eV}^{2} / \mathrm{c}^{4} .
$$

Using the self consistently fitted $P_{\text {ne }}$ value instead, we have to take into account that its systematic uncertainty of \pm 0.022 is anticorrelated to the corresponding uncertainty of $\mp 0.023$ of $\sigma_{\text {tot }} \rho d$ according to Fig. 18. Their combined action on $m^{2}\left(\nu_{e}\right)$ has to be calculated, therefore. Moreover, we have to consider the statistical uncertainty $\delta P_{\text {ne,stat }}= \pm 0.016$ which results from the fit in Fig. 17 Added in quadrature to the systematic contribution we obtain from the self consistent analysis for the optimum interval $E>18500 \mathrm{eV}$ a combined systematic uncertainty of

$$
\delta m^{2}\left(\nu_{e}\right)_{\text {syst, selfcons. }}\left(P_{\text {ne }}, \sigma_{\text {tot }} \rho d\right)=1.58 \mathrm{eV}^{2} / \mathrm{c}^{4} .
$$

The marginal reduction compared to (29) would not really be worth the effort. Rather we emphasize that it determines for the first time the probability of neighbour excitation from the data themselves and confirms, moreover, the qualitative estimation of correction factors applied earlier to Kolos' original calculation of $P_{\text {ne }}$ 39].

In addition the slight shift of the central value of $P_{\text {ne }}$ from 0.046 in the standard analysis to 0.05 in the selfconsistent one causes a corresponding shift of the $m^{2}\left(\nu_{e}\right)$ fit value of the final result still closer to zero

$$
\begin{array}{r}
m^{2}\left(\nu_{e}\right)=\left(-0.6 \pm 2.2_{\text {stat }} \pm 2.1_{\text {syst }}\right) \mathrm{eV}^{2} / \mathrm{c}^{4} \\
\chi^{2} / \text { d.o.f. }=208 / 194
\end{array}
$$

\section{Discussion of results}

\subsection{Experimental $\beta$ spectrum}

We start the discussion by taking a look at the measured spectra in the vicinity of the endpoint (Fig. [20). The bulk of phase II data has been obtained in runs Q5 to Q8 under almost identical conditions and may hence be composed here to a single spectrum (full squares). The open squares represent runs Q11 and Q12. The rate is slightly higher and the background still lower than in the Q5 to Q8 runs. For comparison we also show the last spectrum from phase I taken in 1994 (open circles) [26 59]. The full curve is a fit to the Q5 to Q8 data with $m^{2}\left(\nu_{e}\right)$ fixed to zero, looking perfect. Already $5 \mathrm{eV}$ below the effective endpoint the spectrum rises distinctly from the background, excluding prima vista any larger neutrino mass. (The shift of the effective endpoint from the true one is obtained from an average over the ro- vibrational excitation of the daughter molecule, over the transmission function, and over the source charging). Moreover, these summed up data exclude safely any steady spectral anomaly close to the endpoint on the level of $1 \mathrm{mHz}$; this corresponds to about $10^{-12}$ of the total decay rate of the source. 


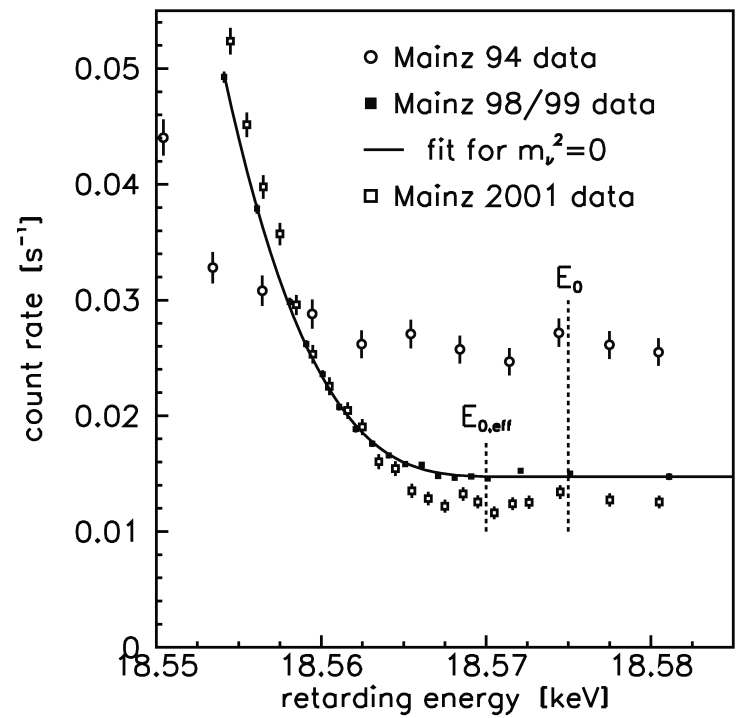

Fig. 20. Averaged count rate of the $98 / 99$ data (filled squares) with fit for $m^{2}\left(\nu_{e}\right)=0$ (line) and the 2001 data (open squares) in comparison with previous Mainz data from phase I (open circles) plotted as function of the retarding potential near the endpoint $E_{0}$.

\subsection{Troitsk anomaly}

The absence of any anomaly in the summed up spectrum does not exclude necessarily a step like Troitsk anomaly which would fluctuate in position and amplitude. It might be washed out in the summed up data. Indeed it has been observed to be a fluctuating effect, for some time hinting to a half year period even 28. In December 2000, however, it appeared as sudden outburst [74]. In this period Q10 was running at Mainz in parallel. Fig. 21] shows the analysis of both runs with respect to the appearance of a step in the integral spectrum, i.e. a line in the original spectrum. To that end one fits the spectrum to the data under the assumption of an additional sharp line of free amplitude at a particular position. The upper plot shows the course of $\chi^{2}$ as function of the line position for the Troitsk data. A very significant minimum is observed at $18553 \mathrm{eV}$ indicating a line, (or step, respectively) with an equally significant amplitude of $13 \mathrm{mHz}$ (middle plot). The corresponding $\chi^{2}$ plot for the parallel run at Mainz with similar sensitivity shows but fluctuations of statistical size (lower plot). Hence speculations that the Troitsk anomaly might be due to a fluctuating presence of dense neutrino clouds [28] are disproved. Rather it has to be attributed to instrumental effects, as pointed out already in section 4.2 (see also [36] and [44]).

\section{$6.3 m^{2}\left(\nu_{e}\right)$ result and upper limit of $m\left(\nu_{e}\right)$}

From the two alternative choices of the neighbour excitation probability we settle on the self-consistently determined one for reasons given in sections 5.6 and 5.7. Hence
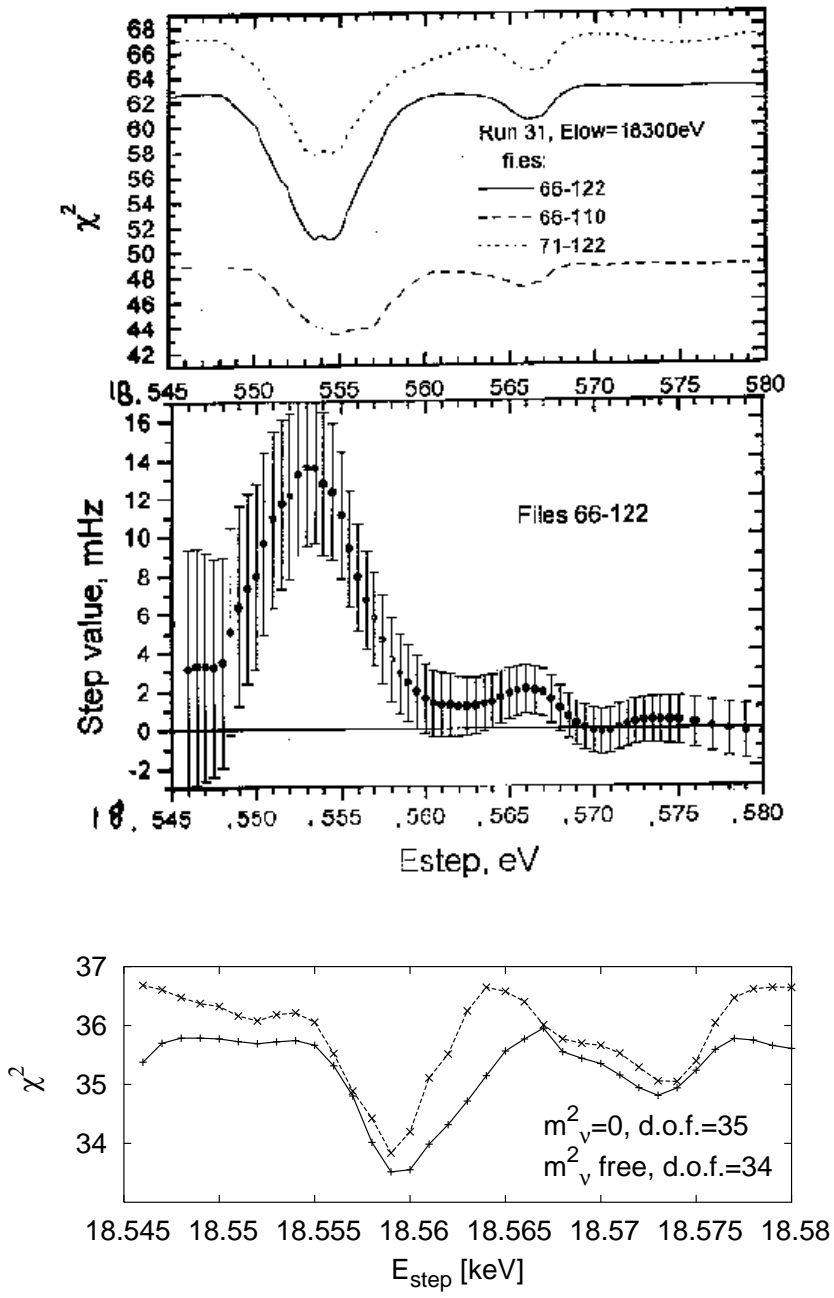

Fig. 21. Search for a step like anomaly in parallel measurements in december 2000. The upper and the middle graph show the analysis of the Troitsk data 74 by adding position and amplitude of the step as free parameters. The upper graph shows the resulting drop of $\chi^{2}$, the lower one the fitted step amplitude, both as function of the step position. A very significant signal appears around $18553 \mathrm{eV}$. In contrast the corresponding $\chi^{2}$ plot for the Mainz data (lower graph) is shown, fluctuations are insignificantly by only 2 units.

(31) is our final experimental result on the observable $m^{2}\left(\nu_{e}\right)$. As compared to our ealier communicated result [29] it has improved in three respects:

1. The statistical uncertainty has been diminished further by $0.3 \mathrm{eV}^{2} / \mathrm{c}^{4}$. 


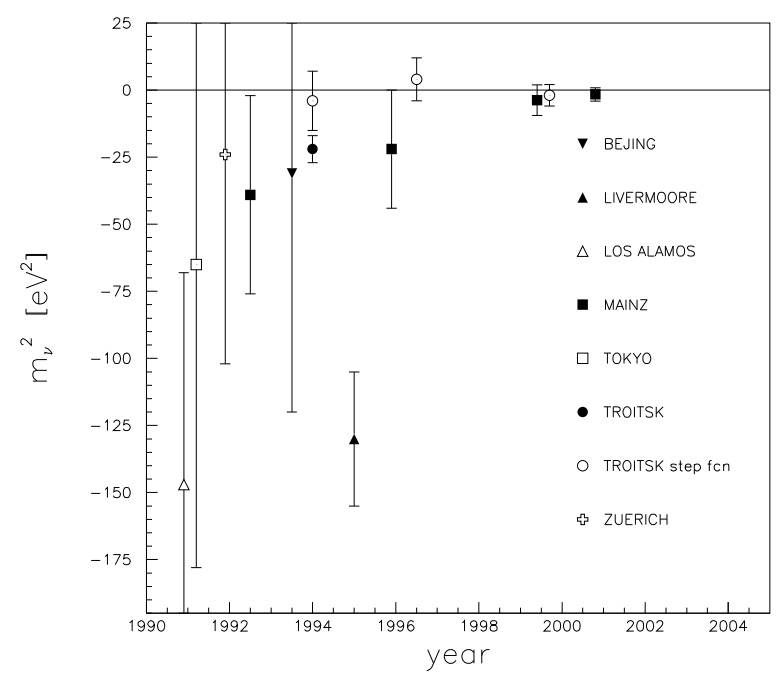

Fig. 22. Published results of squared neutrino mass values $m^{2}\left(\nu_{e}\right)$ obtained from tritium decay since 1990 . The already finished experiments at Los Alamos, Zürich, Tokyo, Beijing and Livermore [76 77 78,79 80] used magnetic spectrometers, the experiments at Troitsk and Mainz are using MAC-E-Filters (as described before).

2. The systematic uncertainty has been better founded with respect to the questionable neighbour excitation probability (but remained unchanged in size).

3. The central value has moved further up from $-1.6 \mathrm{eV}^{2} / \mathrm{c}^{4}$ to $-0.6 \mathrm{eV}^{2} / \mathrm{c}^{4}$ and has lost by now any touch of being unphysical in view of the error bars.

The progress in the observable $m^{2}\left(\nu_{e}\right)$ of this final Mainz result as compared to the most sensitive earlier experiments using momentum analysing spectrometers approaches 2 orders of magnitude (Fig. [22). The Troitsk group communicated similar numbers 75] $\left(m^{2}\left(\nu_{e}\right)=\left(-2.3 \pm 2.5_{\text {stat }} \pm\right.\right.$ $\left.\left.2.0_{\text {syst }}\right) \mathrm{eV}^{2} / \mathrm{c}^{4}\right)$, but there is an important difference. The Troitsk group needs to correct for the observed anomaly by adding phenomenologically a sharp line with free position and size to the $\beta$ spectrum without including a systematic correction for this approach. Without this correction the fit would charge this effect on $m^{2}\left(\nu_{e}\right)$ and drive it negative as discussed above already. Since phenomenology and origin of the anomaly are barely known, this procedure is not obvious and it is difficult to assign a proper systematic uncertainty to this correction. Up to now the Troitsk group has not considered in its result any systematic uncertainty of this correction.

If we move the central value of $m^{2}\left(\nu_{e}\right)$ to zero and calculate from there the upper mass limit at 95\% C.L., then we obtain the so-called sensitivity limit. It lies for both evaluations (28) and (31) at

$$
m_{\text {sens. } \lim .}\left(\nu_{e}\right)=2.4 \mathrm{eV} / \mathrm{c}^{2} \quad(95 \% \text { C.L. }) .
$$

Since the actual $m^{2}\left(\nu_{e}\right)$ values are slightly negative we derive from them an upper limit by help of the unified approach [81], recommended by the particle data group.
This yields in case of the result (28) from the standard analysis

$$
m\left(\nu_{e}\right)<2.2 \mathrm{eV} / \mathrm{c}^{2} \quad(95 \% \text { C.L. })
$$

which agrees with the latest communicated value 29 . Our preferred result (31), however, calculated with a selfconsistent $P_{\text {ne }}$ value, yields a slightly higher limit

$$
m\left(\nu_{e}\right)<2.3 \mathrm{eV} / \mathrm{c}^{2} \quad(95 \% \text { C.L. })
$$

since the respective $m^{2}\left(\nu_{e}\right)$ value lies still closer to zero. The increasing reduction of the upper limit below the sensitivity limit as function of an increasing negative $m^{2}\left(\nu_{e}\right)$ value, is but a dubious gift of the unified approach, accompanied by an increase of the probability that the result suffers from unidentified systematic errors. We quote (34) instead of (32) or (33) as our final upper mass limit, because it stems from a more consistent analysis of the data on one hand and conforms to the recommended unified approach on the other. Anyway, their difference is marginal.

\section{Conclusion and outlook}

Phase II of the Mainz neutrino mass experiment started in 1995 with substantial improvements regarding the frozen $\mathrm{T}_{2}$ source as well as background rejection in the $\beta$ transport channel and in the electrostatic spectrometer. This enabled running at a 10 times better signal to background ratio from 1997 on. Thereafter, a number of side experiments yielded:

1. A detailed study and suppression of the unexpected and disturbing dewetting of the $\mathrm{T}_{2}$ film from the substrate [46 47].

2. The discovery, quantification and theoretical explanation of source charging [1].

3. A determination of the energy loss spectrum of $\beta$ particles in solid $\mathrm{T}_{2}$ 48.

4. Phenomenological studies and suppression of background mechanisms in MAC-E Filters.

They formed the basis for a satisfactory control and reduction of systematic uncertainties in parallel to the statistical one.

Data taking on the search for the neutrino mass covered the years 1998 to 2001 and yielded the so far narrowest limit on the observable $m^{2}\left(\nu_{e}\right)$ of $(-0.6 \pm 3.0) \mathrm{eV}^{2} / \mathrm{c}^{4}$ from which an upper limit $m\left(\nu_{e}\right)<2.3 \mathrm{eV} / \mathrm{c}^{2}$ (90\% C.L.) is derived.

The discovery of neutrino oscillations at squared mass differences of $\Delta m_{i j}^{2} \leq 0.05 \mathrm{eV}^{2} / \mathrm{c}^{4}$ [1,2,3,4, 5, 6, 7, 8, 9, 10] allows furthermore to apply the same upper limit to all three neutrino flavours as reference value in particle and astrophysics. Clearly there is a burning interest to improve this limit further in order to check cosmological models more sensitively by laboratory results on one hand and to decide the alternative between degenerate and hierarchical neutrino masses on the other. In this respect a recent paper on the final data from the Heidelberg Moscow 
experiment is giving a first indication; it reports on a $4 \sigma$ signal of neutrinoless double $\beta$ decay in ${ }^{76} \mathrm{Ge}$ [12]. Explained by an exchange of a massive Majorana neutrino, this signal would give a mass in approximate limits $0.1 \leq m_{e e} \mathrm{c}^{2} / \mathrm{eV} \leq 0.9$ (95\% C.L.).

Obviously our present experiment has exhausted its potential by now, almost 20 years after it has been first conceived. First plans to build either a rigorously enlarged MAC-E-Filter 70, or a bent variant offering in addition a highly resolving differential energy analysis 82 were proposed at an Erice meeting in 1997. A following paper treated in some detail the potential of a MAC-E-Filter of $5 \mathrm{~m}$ diameter and reported moreover on an implementation of a time of flight mode which transforms a MACE-Filter from a high pass to a narrow band filter with equally sharp slopes 68. In 2000 the KATRIN collaboration [57] formed proposing to build a large MAC-E-filter in combination with a gaseous $\mathrm{T}_{2}$ source at the site of the Forschungszentrum Karlsruhe. It combines the expertise from the foregoing experiments at Los Alamos [72, Mainz and Troitsk with the strength of a national laboratory including expertise in handling large amounts of tritium. The present design aims at reaching within 3 years of measurement a precision of $\Delta m^{2}\left(\nu_{e}\right) \approx 0.02 \mathrm{eV}^{2} / \mathrm{c}^{4}$ corresponding to a sensitivity limit of $0.2 \mathrm{eV} / \mathrm{c}^{2}$ for the mass itself. The experiment should be ready to go in 2008 .

\section{Acknowledgement}

The authors are grateful to the Deutsche Forschungsgemeinschaft, which supported this experiment under contract no. Ot33 from its beginning until 2000 and to the Bundesministerium für Bildung und Wissenschaft which took over thereafter under contract no. 06Mz866I/5. Our thanks also go to our frequent guests from Troitsk, V. Lobashev (supported by an Alexander von Humboldt award) as well as O. Kazachenko and N. Titov (supported by a visitor programme of the Deutsche Forschungsgemeinschaft) for their valuable contributions.

\section{References}

1. S. Fukuda and the Superkamiokande Collaboration, Phys. Rev. Lett. 81, 1562 (1998)

2. R. Davis, Nucl. Phys. B (Proc. Suppl.) 48, 284 (1996)

3. M.B. Smy and the Superkamiokande Collaboration, Nucl. Phys. B (Proc. Suppl.) 118, 25 (2003)

4. B.T. Cleveland et al., Astrophys. J. 496, 505, (1998)

5. T.A. Kirsten and the GNO Collaboration, Nucl. Phys. B

(Proc. Suppl.) 118, 33 (2003)

6. V.N. Garvin and the SAGE Collaboration, Nucl. Phys. B (Proc. Suppl.) 118, 39 (2003)

7. A.L. Hallin and the SNO Collaboration, Nucl. Phys. B (Proc. Suppl.) 118, 3 (2003)

8. Q.R. Ahmad et al., Phys. Rev. Lett. 89, 011301, (2002)

9. K. Eguchi and the KamLAND Collaboration, Phys. Rev. Lett. 90, 021802 (2003)

10. M.C. Gonzalez-Garcia, Y. Nir, Rev. Mod. Phys. 75, 345 (2003)
11. H.V. Klapdor-Kleingrothaus, A. Dietz, H.L. Harney, I.V. Krivosheina, Mod. Phys. Lett. A16, 2409 (2001)

12. H.V. Klapdor-Kleingrothaus, I.V. Krivosheina, A. Dietz, O. Chkvorets, Phys. Lett. B586, 198 (2004)

13. D.N. Spergel et al., arXiv:astro-ph/0302209 (2003), Astrophys. J. (Suppl.) 148, 175 (2003)

14. S. Hannestad, JCAP 0305, 004 (2003), arXiv: astro-ph/ 0303076 (2003)

15. V. Barger, D. Marfatia, A. Regre, arXiv: hep-ph/0312065 v2 (2003)

16. S.W. Allen, A.C. Fabian, S.L. Fridle, arXiv: astro-ph/0306386 (2003)

17. R.G.H. Robertson, D.A. Knapp, Ann. Rev. Nucl. Sci. 38, 185 (1988)

18. E. Holzschuh, Rep. Proc. Phys. 55, 1035 (1992)

19. J.F. Wilkerson and R.G.H. Robertson in "Current Aspects Of Neutrino Physics", edited by D.O. Caldwell (Springer, Berlin, Heidelberg, 2001), p. 39

20. Ch. Weinheimer in "Neutrino Mass", edited by G. Altarelli and K. Winter, Springer Tracts in Modern Physics 190, (Springer-Verlag, Berlin, Heidelberg, Germany, 2003), p. 25

21. A. Picard, H. Backe, H. Barth, J. Bonn, B. Degen, Th. Edling, R. Haid, A. Hermanni, P. Leiderer, Th. Loeken, A. Molz, R.B. Moore, A. Osipowicz, E.W. Otten, M. Przyrembel, M. Steininger, Ch. Weinheimer, Nucl. Instr. Meth. B 63, 345 (1992)

22. V.M. Lobashev, P.E. Spivac, Phys. Lett. B 460, 3305 (1985)

23. A.I. Belesev et al., Phys. Lett. B 350, 263 (1995)

24. Christian Weinheimer, Doct. Thesis, Mainz, Univ. (1993)

25. Ch. Weinheimer, M. Przyrembel, H. Backe, H. Barth, J. Bonn, B. Degen, Th. Edling, H. Fischer, L. Fleischmann, J.U. Grooß, R. Haid, A. Hermanni, G. Kube, P. Leiderer, Th. Loeken, A. Molz, R.B. Moore, A. Osipowicz, E.W. Otten, A. Picard, M. Schrader, M. Steininger, Phys. Lett. B 300, 210 (1993)

26. H. Backe, H. Barth, A. Beile, J. Bonn, B. Degen, L. Fleischmann, M. Gundlach, E.W. Otten, M. Przyrembel, Ch. Weinheimer in Proc. 17. Int. Conf. on Neutrino Physics and Astrophysics, Helsinki, Finnland, June 1996, World Scientific, Singapore, p. 259

27. Ch. Weinheimer, B. Degen, A. Bleile, J. Bonn, L. Bornschein, O. Kazachenko, A. Kovalik, E.W. Otten, Phys. Lett. B 460, 219 (1999)

28. V.M. Lobashev et al., Phys. Lett. B460, 227 (1999)

29. J. Bonn, B. Bornschein, L. Bornschein, L. Fickinger, B. Flatt, O. Kazachenko, A. Kovalik, Ch. Kraus, E.W. Otten, J.P. Schall, H. Ulrich, Ch. Weinheimer, Nucl. Phys. B (Proc. Suppl.) 91, 273, (2001)

30. R.S. Van Dyck, Jr., D.L. Farnham, P.B. Schwinberg, Phys. Rev. Lett 70, 2888 (1993)

31. J.J. Simpson, Phys. Rev. D 23, 64 (1981)

32. W.W Repco, C.E. Wu, Phys. Rev. C 28, 2433 (1983)

33. S. Gardner, V. Bernard, U.-G. Meißner, Phys. Lett. B 598, 188 (2004)

34. G.J. Stephenson, T. Goldman, Phys. Lett. B 440, 89 (1998)

35. Particle Data Group, Phys. Rev. D 66, 321 (2002)

36. Christine Kraus, Diploma Thesis, Mainz Univ. (2000)

37. O. Fackler, B. Jeziorski, W. Kolos, H.J. Monkhorst, K. Szalewicz, Phys. Rev. Lett. 55, 1388 (1985)

38. A. Saenz, S. Jonsell, P. Froehlich, Phys. Rev. Lett. 84, 242 (2000) 
39. W. Kolos et al., Phys. Rev. A 37, 2297 (1988)

40. E.W. Otten, Prog. Part. Nucl. Phys. 32, 153 (1994)

41. A. Picard, H. Backe, J. Bonn, B. Degen, R. Haid, A. Hermanni, P. Leiderer, A. Osipowicz, E.W. Otten, M. Przyrembel, M. Schrader, M. Steininger, Ch. Weinheimer, Z.Phys. A 342, 71 (1992)

42. Beate Bornschein, Doct. Thesis, Mainz, Univ. (2000)

43. Lutz Bornschein, Doct. Thesis, Mainz, Univ. (2002)

44. Christine Kraus, Doct. Thesis, Mainz, Univ. (2004)

45. Lars Fleischmann, Doct. Thesis, Mainz, Univ. (1998)

46. L. Fleischmann, J. Bonn, B. Degen, M. Przyrembel, E.W. Otten, Ch. Weinheimer, P. Leiderer, J. Low Temp. Phys. 119, 615 (2000)

47. L. Fleischmann, J. Bonn, B. Bornschein, P. Leiderer, M. Przyrembel, Ch. Weinheimer, A. Saenz, Euro. Phys. J. B 16, 521 (2000)

48. V.N. Aseev, A.I. Belesev, A.I. Berlev, E.V. Geraskin, O.V. Kazachenko, Yu.E. Kuznetsov, V.M. Lobashev, R.P. Ostroumov, N.A. Titov, S.V. Zadorozhny, Yu.I. Zakharov, J. Bonn, B. Bornschein, L. Bornschein, E.W. Otten, M. Przyrembel, Ch. Weinheimer, A. Saenz, Euro. Phys. J. D 10, 39 (2000)

49. R. Conradt, U. Albrecht. S. Herminghaus, P. Leiderer, Physica B 194-196, 679 (1994)

50. Oliver Kettig, Dipl. Thesis, Mainz, Univ. (1994)

51. R.M.A. Azzam, N.M. Bashara, Ellipsometry and polarized light, North Holland Publ. Comp, Amsterdam, Netherlands (1988)

52. W. Kolos, L. Wolniewicz, J. Chem. Phys. 46, 1426 (1967)

53. I. Silvera, Rev. Mod. Phys. 52, 393 (1980)

54. Hermann Fischer, Dipl. Thesis, Mainz, Univ. (1980)

55. Herbert Barth, Dipl. Thesis, Mainz, Univ. (1991)

56. Daphne Goldmann, Dipl. Thesis, Mainz, Univ. (1995)

57. A. Osipowicz et al., arXiv:hep-ex/0109033

58. V.M. Lobashev, Troitsk, priv. commun.

59. Herbert Barth, Doct. Thesis, Mainz, Univ. (1997)

60. Ch. Weinheimer, M. Schrader, J. Bonn, Th. Loeken,

H. Backe, Nucl. Instrum. Methods A 311, 273 (1993)

61. Holger Ulrich, Dipl. Thesis, Mainz, Univ. (2000)

62. Jean-Pierre Schall, Dipl. Thesis, Mainz, Univ. (2001)

63. Beatrix Müller, Dipl. Thesis, Mainz, Univ. (2002)

64. Frank Schwamm, Doct. Thesis, Karlsruhe, Univ. (2004)

65. Thomas Thümmler, Dipl. Thesis, Mainz, Univ. (2002)

66. Salvadore Sanchez, Dipl. Thesis, Mainz Univ. (2003)

67. Björn Flatt et al., to be published

68. J. Bonn, L. Bornschein, B. Degen, E.W. Otten, Ch. Weinheimer, NIM A421, 256 (1999)

69. J. Ciborowski, J. Rembielinski, Eur. Phys. C 8, 157 (1999)

70. H. Barth, L. Bornschein, B. Degen, L. Fleischmann, M. Przyrembel, H. Backe, A. Bleile, J. Bonn, D. Goldmann, M. Gundlach, O. Kettig, E.W. Otten, G. Tietze, Ch. Weinheimer, P. Leiderer, O. Kazachenko, A. Kovalik, Prog. Part. Nucl. Phys. 40, 353 (1998)

71. B. Bornschein, J. Bonn, L. Bornschein, E.W. Otten, Ch. Weinheimer, Journal of Low Temperature Physics, 131, 69 (2003)

72. J.F. Wilkerson et al., Phys. Rev. Lett. 58, 2023 (1987)

73. A. Saenz, Humbold Univ., Berlin, priv. commun.

74. V.M. Lobashev, talk and proc. Int. Conf. on NonAccelerator New Physics, Dubna, 2001

75. V.M. Lobashev, Proc. 17. Int. Conf. on Nuclear Physics in Astrophysics, Debrecen/Hungary, 2002, Nucl. Phys. A719 (2003) 153c-160c
76. R.G.H. Robertson et al., Phys. Rev. Lett. 87, 957 (1991)

77. E. Holzschuh et al., Phys. Lett. B287, 381 (1992)

78. H. Kawakami et al., Phys. Lett. B256, 105 (1991)

79. H.C. Sun et al., CJNP 15, 261 (1993)

80. W. Stoeffl, D.J. Decman, Phys. Rev. Lett. 75, 3237 (1995)

81. G.J. Feldmann and R.D. Cousins, Phys. Rev. D57, 3873 (1998)

82. V.M. Lobashev, Prog. Port, Nucl. Phys. 40, 337 (1998) 